\title{
ON DETERMINING UNKNOWN FUNCTIONS IN DIFFERENTIAL SYSTEMS, WITH AN APPLICATION TO BIOLOGICAL REACTORS.
}

\author{
Éric Busvelle ${ }^{1}$ AND JeAn-Paul Gauthier ${ }^{1}$
}

\begin{abstract}
In this paper, we consider general nonlinear systems with observations, containing a (single) unknown function $\varphi$. We study the possibility to learn about this unknown function via the observations: if it is possible to determine the [values of the] unknown function from any experiment [on the set of states visited during the experiment], and for any arbitrary input function, on any time interval, we say that the system is "identifiable". For systems without controls, we give a more or less complete picture of what happens for this identifiability property. This picture is very similar to the picture of the "observation theory" in [7]:

1. if the number of observations is three or more, then, systems are generically identifiable;

2 . if the number of observations is 1 or 2 , then the situation is reversed. Identifiability is not at all generic. In that case, we add a more tractable infinitesimal condition, to define the "infinitesimal identifiability" property. This property is so restrictive, that we can almost characterize it (we can characterize it by geometric properties, on an open-dense subset of the product of the state space $X$ by the set of values of $\varphi$ ). This, surprisingly, leads to a non trivial classification, and to certain corresponding "identifiability normal forms".

Contrarily to the case of the observability property, in order to identify in practice, there is in general no hope to do something better than using "approximate differentiators", as show very elementary examples. However, a practical methodology is proposed in some cases. It shows very reasonable performances.

As an illustration of what may happen in controlled cases, we consider the equations of a biological reactor, $[2,4]$, in which a population is fed by some substrate. The model heavily depends on a "growth function", expressing the way the population grows in presence of the substrate. The problem is to identify this "growth function". We give several identifiability results, and identification methods, adapted to this problem.
\end{abstract}

Mathematics Subject Classification. 93B07, 93B10, 93B30, 78A70.

Received June 12, 2002. Revised January 14 and March 23, 2003.

\section{INTRODUCTION, MAIN RESUlts, ORGANIZATION OF THE PAPER}

\subsection{Systems under consideration}

In this paper, depending on the context, smooth will mean $C^{\omega}$ (real-analytic), or $C^{\infty}$, or $C^{k}$, for an integer $k$ large enough.

Keywords and phrases. Nonlinear systems, observability, identifiability, identification.

${ }^{1}$ Laboratoire d'Analyse Appliquée et Optimisation, Département de Mathématiques, Université de Bourgogne,

bâtiment Mirande, BP. 47870, 21078 Dijon Cedex, France; e-mail: busvelle@u-bourgogne.fr, gauthier@u-bourgogne.fr 
Very often, in nonlinear control systems, certain special variables with "physical meaning" appear, (called here the "internal variables") and the system depends on certain functions of these variables. These functions describe some physical characteristic inside the system, and it may happen that they are not well known, and have to be determined on the basis of experiments.

We consider general smooth nonlinear systems:

$$
\Sigma:\left\{\begin{array}{l}
\frac{\mathrm{d} x}{\mathrm{~d} t}=f(x, u(t), \varphi \circ \pi(x(t))) ; \\
y=h(x, u(t), \varphi \circ \pi(x(t))),
\end{array}\right.
$$

or such systems that are "uncontrolled":

$$
\begin{aligned}
\frac{\mathrm{d} x}{\mathrm{~d} t} & =f(x, \varphi \circ \pi(x(t))) ; \\
y & =h(x, \varphi \circ \pi(x(t))),
\end{aligned}
$$

where $x \in X$ denotes the state, $y$ denotes the observation, $u(\cdot)$ is the control function, $z=\pi(x)$ is the "internal variable", $\pi$ is called the internal mapping, $\pi: X \rightarrow Z$. We assume that $X$ and $Z$ are given analytic connected manifolds, both Hausdorf and paracompact. Here $\operatorname{dim}(X)=n$.

The (smooth) "unknown function" is denoted by $\varphi: Z \rightarrow I$. Here, $I$ will denote a compact interval of $\mathbb{R}$. To finish, $y \in \mathbb{R}^{d_{y}}, u \in U \subset \mathbb{R}^{d_{u}}$, where $U$ is a compact subanalytic subset of $\mathbb{R}^{d_{u}}$. Also, $f$ is a $(u, \varphi)$-parametrized smooth vector field, The observation mapping, $h$, is a smooth mapping: $X \times U \times I \rightarrow \mathbb{R}^{d_{y}}$.

In the following, the systems $\Sigma=(f, h)$ will vary, but the manifolds $X, Z$, the (smooth) internal mapping $\pi$, the space $\mathbb{R}^{d_{y}}$, and the sets $I$ and $U$ are given and fixed.

\subsection{Rough definitions and statement of the main theoretical results}

Our main results in this paper are about uncontrolled systems. For these systems, we provide an almost complete theory of identifiability. Controlled systems may have very different identifiability properties. Just to show what may happen for controlled systems, we will treat a practical example: the model of a "biological reactor". In fact, the general study in this paper was originally motivated by this very interesting elementary example.

\subsubsection{Definitions}

Roughly speaking, we say that a system is identifiable if, for each experiment (input-output trajectory), one can reconstruct the piece (visited during the experiment) of the graph of the unknown function, on the basis of the input-output data.

Given any trajectory of the system:

$$
\mathcal{T}=\{(u(t), \varphi \circ \pi(x(t))=\hat{\varphi}(t), x(t), y(t))\},
$$

defined on some time interval $[0, T]$, consider $\hat{\varphi}(t)$ as an (unknown) extra input of the system. The control function $u(\cdot)$ being fixed, we have a well defined mapping:

$$
P_{\Sigma, u(\cdot)}:\left(\hat{\varphi}(\cdot), x_{0}\right) \rightarrow y(\cdot),
$$

where $x_{0}$ is the initial condition for the system. Then, identifiability of $\Sigma$ means that all these mappings (depending on $u(\cdot)$ ) are injective.

Our approach to the analysis of the identifiability property is very similar to the approach in [7], for the analysis of the observability property. The results we obtain are in the same spirit. 
The injectivity of maps being not a stable property, it is not easy to handle. Hence it is reasonable to add to the injectivity requirement the additional requirement of "infinitesimal injectivity" (i.e. injectivity of all the tangent mappings $T P_{\Sigma, u(\cdot)}$ ), to get a more tractable notion of identifiability. This notion is called uniform infinitesimal identifiability.

We denote by $j^{k} y=\left(y(0), y^{\prime}(0), \ldots, y^{(k-1)}(0)\right)$, the $k$-jet at $t=0$ of a smooth function $y(t)$. Another way to manage with the identifiability property is to consider the notion of differential identifiability, i.e. identifiability at the level of the $k$-jets. For this, consider the mappings:

$$
j^{k} P_{\Sigma, u(\cdot)}:\left(j^{k} \hat{\varphi}, x_{0}\right) \rightarrow j^{k} y .
$$

The system is said differentially identifiable (of order $k$ ), if:

$$
j^{k} y^{1}=j^{k} y^{2} \Longrightarrow\left(\hat{\varphi}^{1}(0), x_{0}^{1}\right)=\left(\hat{\varphi}^{2}(0), x_{0}^{2}\right) .
$$

For uncontrolled systems, $k$-differential identifiability implies identifiability, for all smooth internal mappings $\pi$. (For controlled systems, the situation is much more complicated. We refer to [7] for similar questions in the context of the observability property.)

Precise definitions of identifiability, uniform infinitesimal identifiability, and differential identifiability are given and discussed in Section 2.1.

In the remaining of this section, we consider the "uncontrolled case" only, i.e. there is no control variable $u(t)$. In that case, we will get a lot of general results. To state them, let us endow the set $S$ of uncontrolled systems, of the form (1.2) with the $C^{\infty}$ Whitney topology ${ }^{2}$.

\subsubsection{Results for the generic case}

In Section 3, we will show the following important result:

Theorem 1. If the number of outputs is larger or equal to 3 , then, differential identifiability of order $2 n+1$ $(n=\operatorname{dim} X)$, is a generic property. This is true in the Baire sense only (i.e. the set of differentially identifiable systems is residual). This implies in particular that identifiability is a generic property.

Remark 1. This theorem has to be compared to the corresponding result for the observability theory (see $[6,7])$. In the case of "single input observability", the number of outputs has to be larger or equal to 2 only. If one thinks about the function to be identified, as a control variable (which is what we do here in), it is surprising that the number of outputs for generic identifiability be 3 . At a first glance, it should be 2 .

In Section 3, we will show an open set of systems with 2 outputs, which is not identifiable. It is known [7] that single input, single output generic systems are already not observable. Hence, identifiability is not a generic property in the uncontrolled single output case.

\subsubsection{Results for the single output case.}

This case will be the subject of Section 4.

In that case, by the previous section, differential identifiability is not generic. Again similarly to the observability theory of $[5,7]$, we will consider the uniform infinitesimal identifiability property. It is a so restrictive property (infinite codimension, in fact) that it can be completely characterized.

We will show the following theorem, in the analytic case (i.e. the system is $C^{\omega}$ ).

\footnotetext{
${ }^{2}$ Consider subsets $V_{j}$ of the vector space $S^{j}$ of $C^{j}$ functions (resp. vector fields, ...) over a $C^{\infty}$ manifold $X$, of the following form: $V_{j}=\cap_{\alpha \in A} V_{\alpha}$, such that there is a locally finite family of compact sets $\left\{U_{\alpha}, \alpha \in A\right\}$, where $V_{\alpha}$ is a neighborhood of zero in the topology (non Hausdorf unless $U_{\alpha}=X$ ) of uniform $C^{j}$ convergence over $U_{\alpha}$. Sets of the form $V_{j}$ are a basis of neighborhoods of zero of the $C^{j}$ Whitney topology over $S^{j}, 0 \leq j<\infty$. This topology is not metrizable (unless $X$ is compact), nevertheless, it is Baire. The $C^{\infty}$ Whitney topology is the union of the topologies induced on $S^{\infty}$ by the $C^{j}$ Whitney topologies over $S^{j}$ via the inclusions $S^{\infty} \hookrightarrow S^{j}$. See [11] for details.
} 
In the following, $\operatorname{dim}(X)=n$, and $L_{f}$ is the Lie-derivative operator on $X$. Also, $f_{\varphi}$ denotes the vector field $f(., \varphi)$, for $\varphi \in I$, and $h_{\varphi}: X \rightarrow R^{d_{y}}$ is the map $h(., \varphi)$. The symbol $d_{x}$ means differential with respect to $x$ only.

Theorem 2. If $\Sigma$ is uniformly infinitesimally identifiable, then, there is a subanalytic closed subset $Z$ of $X$, of codimension 1 at least, such that on the open set $X \backslash Z$, the following two equivalent properties 1 and 2 below hold:

1. a. $\frac{\partial}{\partial \varphi}\left\{\left(L_{f_{\varphi}}\right)^{k} h_{\varphi} \equiv 0\right.$, for $k=0, \ldots, n-1, b . \frac{\partial}{\partial \varphi}\left\{\left(L_{f_{\varphi}}\right)^{n} h_{\varphi} \neq 0\right.$ (in the sense that it never vanishes), c. $d_{x} h_{\varphi} \wedge \ldots \wedge d_{x} L_{f_{\varphi}}^{n-1} h_{\varphi} \neq 0$;

2. any $x_{0} \in X \backslash Z$ has a coordinate neighborhood $\left(x_{1}, \ldots, x_{n}, V_{x_{0}}\right), V_{x_{0}} \subset X \backslash Z$ in which $\Sigma$ (restricted to $\left.V_{x_{0}}\right)$ can be written:

$$
\left\{\begin{array}{c}
\dot{x}_{1}=x_{2}, \\
\dot{x}_{2}=x_{3}, \\
\cdot \\
\cdot \\
\dot{x}_{n-1}=x_{n} \\
\dot{x}_{n}=\psi(x, \varphi) \\
y=x_{1} ;
\end{array}\right.
$$

where $\frac{\partial}{\partial \varphi} \psi(x, \varphi)$ never vanishes.

This theorem has the following pseudo-converse:

Theorem 3. Assume that $\Sigma$ meets the equivalent conditions of the previous theorem.

Then, any $x_{0}$ has a neighborhood $V_{x_{0}}$ such that the restriction $\Sigma_{\mid V_{x_{0}}}$ of $\Sigma$ to $V_{x_{0}}$ is uniformly infinitesimally identifiable, identifiable and differentially identifiable of order $n+1$.

Notice that Theorem 2 has a global character: it is almost everywhere on $X$, but it is global with respect to $\varphi \in I$.

\subsubsection{The 2-output case}

This case, for analytic systems, will be the purpose of Section 5 .

Again, (differential) "identifiability" being not generic, we will consider the "uniform infinitesimal identifiability" property, that is very restrictive (infinite codimension), so that we will be able to characterize it completely in a geometric way.

Since this geometric description is non obvious, and since there is (surprisingly) a small but nontrivial zoology (3 distinct cases), we do not give the intrinsic geometric characterization here. This is done in Section 5 (Th. 9). In this introduction, we state an equivalent theorem (Th. 4) in terms of normal forms.

Theorem 4. If $\Sigma$ is uniformly infinitesimally identifiable, then, there is an open-dense semi-analytic subset $\tilde{U}$ of $X \times I$, such that each point $\left(x_{0}, \varphi_{0}\right)$ of $\tilde{U}$, has a neighborhood $V_{x_{0}} \times I_{\varphi_{0}}$, and coordinates $x$ on $V_{x_{0}}$ such that 
the system $\Sigma$ restricted to $V_{x_{0}} \times I_{\varphi_{0}}$, denoted by $\Sigma_{\mid V_{x_{0}} \times I_{\varphi_{0}}}$, has one of the three following normal forms:

- type 1 normal form: (in that case, $n>2 k$ )

$$
\begin{aligned}
y_{1} & =x_{1}, y_{2}=x_{2}, \\
\dot{x}_{1} & =x_{3}, \dot{x}_{2}=x_{4}, \\
& \ldots \\
\dot{x}_{2 k-3} & =x_{2 k-1}, \dot{x}_{2 k-2}=x_{2 k}, \\
\dot{x}_{2 k-1} & =f_{2 k-1}\left(x_{1}, \ldots, x_{2 k+1}\right), \\
\dot{x}_{2 k} & =x_{2 k+1}, \\
& \ldots \\
\dot{x}_{n-1} & =x_{n}, \\
\dot{x}_{n} & =f_{n}(x, \varphi), \text { with } \frac{\partial f_{n}}{\partial \varphi} \neq 0 \text { (never vanishes), }
\end{aligned}
$$

- type 2 normal form:

$$
\begin{aligned}
y_{1} & =x_{1}, y_{2}=x_{2}, \\
\dot{x}_{1} & =x_{3}, \dot{x}_{2}=x_{4}, \\
& \ldots \\
\dot{x}_{2 r-3} & =x_{2 r-1}, \dot{x}_{2 r-2}=x_{2 r}, \\
\dot{x}_{2 r-1} & =\Phi(x, \varphi), \dot{x}_{2 r}=F_{2 r}\left(x_{1}, \ldots, x_{2 r+1}, \Phi(x, \varphi)\right), \\
\dot{x}_{2 r+1} & =F_{2 r+1}\left(x_{1}, \ldots, x_{2 r+2}, \Phi(x, \varphi)\right), \\
& \ldots \\
\dot{x}_{n-1} & =F_{n-1}(x, \Phi(x, \varphi)), \\
\dot{x}_{n} & =F_{n}(x, \varphi),
\end{aligned}
$$

with $\frac{\partial \Phi}{\partial \varphi} \neq 0, \frac{\partial F_{2 r}}{\partial x_{2 r+1}} \neq 0$ (hence $\left.n>2 r\right), \ldots, \frac{\partial F_{n-1}}{\partial x_{n}} \neq 0$,

- type 3 normal form:

$$
\begin{aligned}
y_{1} & =x_{1}, y_{2}=x_{2}, \\
\dot{x}_{1} & =x_{3}, \dot{x}_{2}=x_{4}, \\
& \ldots \\
\dot{x}_{n-3} & =x_{n-1}, \dot{x}_{n-2}=x_{n}, \\
\dot{x}_{n-1} & =f_{n-1}(x, \varphi), \dot{x}_{n}=f_{n}(x, \varphi),
\end{aligned}
$$

where $\left(\frac{\partial f_{n-1}}{\partial \varphi}, \frac{\partial f_{n}}{\partial \varphi}\right)$ never vanishes.

Remark 2. a) Among the normal forms in Theorems 2, 4, normal form "type 2" has a special feature: it is close to the uniform infinitesimal observability normal form obtained in [5,7]: one of the outputs $\left(y_{1}\right)$ is used to obtain $\Phi(x, \varphi)$ after successive differentiations. The remaining part of the normal form is exactly (after a number of direct differentiations) the uniform infinitesimal observability normal form, with $\Phi(x, \varphi)$ considered as an input.

b) Type 1 and type 2 normal forms are normal up to a permutation of the two outputs $y_{1}$ and $y_{2}$. 
In Section 5, we will give a lot of complementary results, examples, weak converses of Theorems 4, 9, and a few "global" results.

\subsection{Contents and organization of the paper}

The purpose of the next Section 2 is twofold: first we make clear in details the "rough definitions" of "Identifiability" just introduced. Second, we present our controlled example: the bioreactor. We state our main "identifiability result" for this example.

Sections 3-5 are devoted respectively to the proofs of Theorems $1,2,4$, and to complementary results: weak converses of these theorems, examples, ...

In Section 6, we say a few words about the practical problem of identification, specially in the non generic cases: 1 and 2 outputs. In these cases, a reasonable practical methodology for identification is proposed.

Section 7 is devoted to the proof of our identifiability results for the "biological reactor" (we have several such results, corresponding to different choices for the observed variables). We also present a few numerical results for the biological reactor. Some of these results illustrate the general methodology we propose in Section 6 , some others are specific to the example.

\section{Definitions, example}

\subsection{Precise definitions of identifiability}

Associated to a system $\Sigma$ of the form 1.1 or 1.2 , we consider the "input-output mapping" $P_{\Sigma}$ :

$$
\begin{gathered}
P_{\Sigma}: X \times L^{\infty}[U] \times L^{\infty}[I] \rightarrow L^{\infty}\left[\mathbb{R}^{d_{y}}\right] ; \\
\left(x_{0}, u(\cdot), \hat{\varphi}(\cdot)\right) \rightarrow y(\cdot),
\end{gathered}
$$

where $L^{\infty}[U], L^{\infty}[I], L^{\infty}\left[\mathbb{R}^{d_{y}}\right]$ denote the set of $U$-valued (resp. $I$-valued, $\mathbb{R}^{d_{y}}$-valued) measurable, bounded functions, defined on semi-open interval $\left[0, T_{u}\left[,\left[0, T_{\hat{\varphi}}\left[,\left[0, T_{y}\left[\right.\right.\right.\right.\right.\right.$. The mapping $P_{\Sigma}$ is defined as follows:

For any input $u(\cdot) \in L^{\infty}[U]$, any $\hat{\varphi}(\cdot) \in L^{\infty}[I]$, any $x_{0} \in X$, then, the solution $x(t)$ of the Cauchy problem:

$$
\frac{\mathrm{d} x(t)}{\mathrm{d} t}=f(x(t), u(t), \hat{\varphi}(t)), x(0)=x_{0}
$$

is defined on a maximum semi-open interval $\left[0, e\left(x_{0}, u, \hat{\varphi}\right)\left[\right.\right.$, where $0<e\left(x_{0}, u, \hat{\varphi}\right) \leq \min \left(T_{u}, T_{\hat{\varphi}}\right)$. If $e\left(x_{0}, u, \hat{\varphi}\right)<$ $\min \left(T_{u}, T_{\hat{\varphi}}\right)$, then $e\left(x_{0}, u, \hat{\varphi}\right)$ is the positive escape time of $x_{0}$ for the time dependant vector field $f$ in $(2.1)$. For fixed $u, \hat{\varphi}$, the mapping $x_{0} \rightarrow e\left(x_{0}, u, \hat{\varphi}\right) \in \bar{R}_{*}^{+}$is lower semicontinuous $\left(\bar{R}_{*}^{+}=\{t \mid 0<t \leq \infty\}\right)$.

$P_{\Sigma}\left(x_{0}, u, \hat{\varphi}\right)$ is the function $\hat{y}:\left[0, e\left(x_{0}, u, \hat{\varphi}\right)\left[\rightarrow \mathbb{R}^{d_{y}}\right.\right.$, defined by $\hat{y}(t)=h(x(t), u(t), \hat{\varphi}(t))$.

We say that $(u(\cdot), y(\cdot)) \in L^{\infty}[U] \times L^{\infty}\left[\mathbb{R}^{d_{y}}\right]$, with $T_{u}=T_{y}$, is an "admissible input-output trajectory", if there exits a couple $\left(x_{0}, \hat{\varphi}\right) \in X \times L^{\infty}[I]$, such that $y(t)=P_{\Sigma}\left(x_{0}, u, \hat{\varphi}\right)(t)$ for almost all $t \in\left[0, T_{u}[\right.$ (which means in particular $e\left(x_{0}, u, \hat{\varphi}\right)=T_{u}$ ), and $\hat{\varphi}(t)=\varphi \circ \pi(x(t))$, where $\varphi$ is some smooth function, $\varphi: Z \rightarrow I$. (Of course, this $\varphi$ depends on the input-output trajectory (or the "experiment") $(u(\cdot), y(\cdot))$.)

Note that, as a consequence, if $(u(\cdot), y(\cdot))$ is an admissible i-o trajectory, then, $\hat{\varphi}(\cdot)$ in the definition is in fact at least absolutely continuous. In the uncontrolled case, it is smooth.

We define now the notion of identifiability, already introduced in Section 1.2:

Definition 1. The system $\Sigma$ is said "identifiable at" $(u(\cdot), y(\cdot)) \in L^{\infty}[U] \times L^{\infty}\left[\mathbb{R}^{d_{y}}\right]$, with $T_{u}=T_{y}$, if there is at most a single couple $\left(x_{0}, \hat{\varphi}\right) \in X \times L^{\infty}\left(\left[0, T_{u}[, I)\right.\right.$ such that, for almost all $t \in\left[0, T_{u}[\right.$ :

$$
P_{\Sigma}\left(x_{0}, u, \hat{\varphi}\right)(t)=y(t)
$$

and $\hat{\varphi}(t)=\varphi \circ \pi(x(t))$ for some smooth function $\varphi: Z \rightarrow I$. 
Given a system $\Sigma$, the "identifiability set" of $\Sigma$ is the subset of $L^{\infty}[U] \times L^{\infty}\left[\mathbb{R}^{d_{y}}\right]$ formed by the admissible i-o trajectories $(u(\cdot), y(\cdot))$ at which $\Sigma$ is identifiable. If this set is exactly the set of admissible i-o trajectories, then $\Sigma$ is said "identifiable".

Again, in this definition, the smooth function $\varphi$ depends on the i-o trajectory (or the "experiment") $(u(\cdot), y(\cdot))$. Also, again, $\hat{\varphi}$ in this definition is in fact at least absolutely continuous, and smooth in the uncontrolled case.

Of course, this definition is not very tractable in practice, and, as explained in Section 1.2, we will need a few other definitions.

For arbitrary $k$-jets of smooth functions $\hat{\varphi}, \hat{u}$ at $t=0$,

$$
\begin{gathered}
\hat{\varphi}:[0, \varepsilon[\rightarrow I, \hat{u}:[0, \varepsilon[\rightarrow U, \\
j^{k}(\hat{\varphi})=\left(\hat{\varphi}(0), \hat{\varphi}^{\prime}(0), \ldots, \hat{\varphi}^{(k-1)}(0)\right), j^{k}(\hat{u})=\left(\hat{u}(0), \hat{u}^{\prime}(0), \ldots, \hat{u}^{(k-1)}(0)\right),
\end{gathered}
$$

and for any $x_{0} \in X$, the corresponding $k$-jet $j^{k} \hat{y}=\left(\hat{y}, \hat{y}^{\prime}, \ldots, \hat{y}^{(k-1)}\right)$ is well defined, in such a way that the mapping $\Phi_{k}^{\Sigma}:\left(x_{0}, j^{k}(\hat{u}), j^{k}(\hat{\varphi})\right) \rightarrow j^{k} \hat{y}$ be continuous. $\Phi_{k}^{\Sigma}: D_{k} \Phi=X \times\left(U \times \mathbb{R}^{(k-1) d_{u}}\right) \times\left(I \times R^{k-1}\right) \rightarrow \mathbb{R}^{k d_{y}}$.

Remark 3. This mapping $\Phi_{k}^{\Sigma}$ is smooth with respect to $x_{0}, \hat{\varphi}(0), \hat{u}(0)$, and algebraic with respect to $\hat{\varphi}^{\prime}(0), \ldots$, $\hat{\varphi}^{(k-1)}(0)$ and $\hat{u}^{\prime}(0), \ldots, \hat{u}^{(k-1)}(0)$. Also, $\Phi_{k}^{\Sigma}$ depends only on the $k$-jet $j^{k} \Sigma$ of $\Sigma$.

We define $D_{k} \Phi_{2}^{*}$ as the set of couples $\left(\left(x_{1}, j^{k}(\hat{u}), j^{k}\left(\hat{\varphi}_{1}\right)\right),\left(\left(x_{2}, j^{k}(\hat{u}), j^{k}\left(\hat{\varphi}_{2}\right)\right)=\left(z_{1}, z_{2}\right)\right.\right.$, with $z_{1}=\left(x_{1}, j^{k}(\hat{u})\right.$, $\left.j^{k}\left(\hat{\varphi}_{1}\right)\right) \neq\left(\left(x_{2}, j^{k}(\hat{u}), j^{k}\left(\hat{\varphi}_{2}\right)\right)=z_{2}\right.$.

$\Delta_{k}$ is the diagonal in $\mathbb{R}^{k d_{y}} \times \mathbb{R}^{k d_{y}}$. We denote by $\Phi_{k, 2}^{\Sigma, *}$ the mapping: $D_{k} \Phi_{2}^{*} \rightarrow \mathbb{R}^{k d_{y}} \times \mathbb{R}^{k d_{y}},\left(z_{1}, z_{2}\right) \rightarrow$ $\left(\Phi_{k}^{\Sigma}\left(z_{1}\right), \Phi_{k}^{\Sigma}\left(z_{2}\right)\right)$.

Definition 2. The system $\Sigma$ is said "differentially identifiable" of order $k$ if the mapping $\Phi_{k, 2}^{\Sigma, *}$ has the following property: If

then, $\left(x_{1}, \hat{\varphi}_{1}(0)\right)=\left(x_{2}, \hat{\varphi}_{2}(0)\right)$.

$$
\Phi_{k, 2}^{\Sigma, *}\left(z_{1}, z_{2}\right) \in \Delta_{k}
$$

This means that, for all controls (sufficiently differentiable), all couples (initial state, value of $\varphi$ ) are distinguished between them, by the observations and their $k-1$ first derivatives, whatever the other time derivatives of $\varphi$ are.

Equivalently, one can reconstruct the state and the values of $\varphi$, (at a point $\left(x_{0}, u_{0}\right)$, visited by the system) in terms of the controls and their first derivatives, the outputs and their first derivatives.

Remark 4. One could think that a good definition of differential identifiability of order $k$ is just: the map $\Phi_{k}^{\Sigma}$ is injective. Unfortunately, this property is never generic (for uncontrolled systems), if there is less outputs than states $\left(d_{y} \leq n\right)$.

As stated in Section 1.2.2, Theorem 1, differential identifiability at certain orders $k$ is generic, for $d_{y} \geq 3$ (uncontrolled case).

Following the ideas developed in the book [7], and in the papers [5,6] (in the context of observability), we define now the infinitesimal notion of identifiability. For this purpose, we need an adequate concept of the "linearization" of a system.

The mapping $f: X \times U \times I \rightarrow T X$ induces a partial tangent mapping $T_{x, \varphi} f: T X \times T I \times U \rightarrow T T X$. (Here, $T I \sim I \times \mathbb{R}$.) If $\omega$ denotes the canonical involution ${ }^{3}$ of $T T X$, then, $\omega \circ T_{x, \varphi} f$ defines a parametrized vector field on $T X$, also denoted by $T_{x, \varphi} f$ : it is parametrized by the elements $(u,(\varphi, \eta))$ of $U \times T I$.

${ }^{3}$ If $f: X \rightarrow T X$ is a vector field (i.e. a section), then $T f: T X \rightarrow T T X$ is not a section: for $\xi_{x} \in T_{x} X$, then $\Pi_{T T X}\left(T f\left(\xi_{x}\right)\right)=$ $f(x)$, although it should be equal to $\xi_{x}$. There is a well defined involution $\omega: T T X \rightarrow T T X$, called the "canonical involution" of $T T X$, such that $\omega \circ T f$ is a section of $T T X$. See [1] for details. 
Similarly, the function $h: X \times U \times I \rightarrow R^{d_{y}}$, has a partial differential $d_{x, \varphi} h$, and the linearization of $\Sigma$ (or the first variation of $\Sigma$ ) is the following system on $T X$ :

$$
T \Sigma\left\{\begin{array}{l}
\frac{\mathrm{d} \xi}{\mathrm{d} t}=T_{x, \varphi} f(x, u, \varphi ; \xi, \eta) ; \\
\hat{y}=d_{x, \varphi} h(x, u, \varphi ; \xi, \eta),
\end{array}\right.
$$

where $(\xi, \eta) \in T_{x} X \times T_{\varphi} I$.

Denote by $\Pi$ the canonical projection: $T X \rightarrow X$. If $\xi:\left[0, T_{\xi}[\rightarrow T X\right.$ is a trajectory of $T \Sigma$ for the control $u(\cdot)$, function $\varphi(\cdot)$, and the "variation" $\eta(\cdot)$ (all belonging to $L_{\left[0, T_{\xi}\right]}^{\infty}$ ), then, $\Pi \xi$ is a trajectory of $\Sigma$ corresponding to the same control $u(\cdot)$ and function $\varphi(\cdot)$. Conversely, if $\phi_{\tau}(x, u, \hat{\varphi}):[0, e(x, u, \hat{\varphi})[\rightarrow X$ is a trajectory of $\Sigma$ starting from $x_{0}$, and corresponding to the control $u$ and the function $\hat{\varphi}:\left[0, T_{\hat{\varphi}}[\rightarrow I\right.$, then, for all $0<\tau<e(x, u, \hat{\varphi})$, the map:

$$
(x, \varphi) \rightarrow \phi_{\tau}(x, u, \varphi)
$$

is defined on a neighborhood of $\left(x_{0}, \hat{\varphi}\right)$ in $X \times L^{\infty}\left(\left[0, \tau[, I)\right.\right.$, and is differentiable at $\left(x_{0}, \hat{\varphi}\right)$. This differential $T_{x, \varphi} \phi_{\tau}$ is in the usual sense with respect to $x$, and in the Frechet sense with respect to $\varphi$.

For all $\left(\xi_{0}, \eta\right) \in T_{x_{0}} X \times L^{\infty}([0, \tau[, \mathbb{R})$, for almost all $t \in[0, \tau[$ :

$$
P_{T \Sigma}\left(\xi_{0}, \eta\right)(t)=d_{x, \varphi} h\left(u, \hat{\varphi} ; T_{x, \varphi} \phi_{t}\left(u, \hat{\varphi} ; \xi_{0}, \eta\right), \eta\right)=T_{x, \varphi} P_{\Sigma}\left(\xi_{0}, \eta\right)(t) .
$$

The right-hand side of this equality is the differential of the mapping $P_{\Sigma}(x, u, \hat{\varphi})$ with respect to $(x, \varphi)$, at the point $\left(x_{0}, u, \hat{\varphi}\right)$, taken at $\left(\xi_{0}, \eta\right)$, evaluated at time $t$.

Definition 3. The system $\Sigma$ is called "infinitesimally identifiable" at $\left(x_{0}, u(\cdot), \hat{\varphi}(\cdot)\right) \in X \times L^{\infty}[U] \times L^{\infty}[I]$ if all the linear mappings $P_{T \Sigma}: T_{x_{0}} X \times L_{([0, \tau], \mathbb{R})}^{\infty} \rightarrow L_{\left([0, \tau], \mathbb{R}^{d} y\right)}^{\infty}$ in $(2.3)$ (for all $0<\tau<e\left(x_{0}, u, \hat{\varphi}\right)$ ), are injective. It is called "uniformly infinitesimally identifiable", if it is infinitesimally identifiable at all $\left(x_{0}, u(\cdot)\right.$, $\hat{\varphi}(\cdot)) \in X \times L^{\infty}[U] \times L^{\infty}[I]$.

Remark 5. The Definitions 2, 3, do not depend on the internal variables and the internal mapping. On the contrary, Definition 1 does.

A clear comparison between our 3 definitions of identifiability is not obvious at all at the level of this introduction. Definition 1 is a natural and general definition. Definitions 2, 3, are adapted respectively to the generic and non generic cases (uncontrolled), as we shall see.

It will be shown (Th. 7, Sect. 3) that, for uncontrolled systems, differential identifiability at some order $k$ implies identifiability, whatever the internal mapping $\pi$. Also, uniform infinitesimal identifiability implies identifiability of the restrictions of the system to certain open subsets. This last point will be proved in Sections 4, 5 .

Very clearly, our identifiability properties are related to the notion of "left-invertibility", introduced by Hirschorn in the papers [12,13]. One may also refer to the nice survey paper [15], specially Theorem 5.1 (pp. 164-165). In fact, Identifiability is a stronger property than left-invertibility, even for linear single inputoutput systems: left invertibility (in our uncontrolled case) is the injectivity of the maps $\hat{\varphi} \rightarrow P_{\Sigma}(\hat{\varphi})$, for $x_{0}$ chosen and fixed. This injectivity is required at the level of $n$-jets, and locally (which means locally around a fixed $n$-jet of $\hat{\varphi}$, and at all points of an open dense semi-analytic subset of the space of $\left(x_{0}, j^{n} \hat{\varphi}\right)$. If a system is left invertible, roughly speaking, there is an "inverse system", that allows (except at some singularities, and locally), to recover the input of $\Sigma$, from the extension to $n$-jets of its output trajectory. Hence, note that, if people want to practically invert a system, they implicitly consider differentiation of the output. This is connected to the considerations in our Section 6 below.

Obviously, in our (single input) case, invertibility as defined in these papers is generic, and moreover the set of noninvertible systems has infinite codimension. 


\subsection{A comment on these definitions}

Let us limit ourselves to the uncontrolled case in this discussion.

We have 3 definitions (Identifiability, differential identifiability, Uniform Infinitesimal Identifiability). In all these definitions, the functions $\hat{\varphi}(\cdot)$ (or their jets) are considered as extra inputs for the system. These functions have to be smooth in two cases:

a. for Differential Identifiability, since the jets are jets of smooth functions;

b. in the definition of Identifiability, as a consequence of the definition;

But, in Definition 3, of Uniform Infinitesimal Identifiability, no smoothness for $\hat{\varphi}$ and its "variations" $\eta$ is required (we assumed these functions to be in $L^{\infty}$ only, which is natural, as control functions for a nonlinear system). One could think that enlarging in this way the class of "inputs" $\hat{\varphi}$ might restrict a lot the class of systems that are uniformly infinitesimally identifiable.

In fact, the class of $\hat{\varphi}$ and its "variations" $\eta$ plays no role in this definition. This can be seen as a consequence of two distinct facts:

1. first, the proofs of our uniform infinitesimal identifiability results are all based upon a contradiction with the existence of smooth couples $(\hat{\varphi}, \eta)$, that make the system non infinitesimally identifiable along corresponding trajectories. Then, we could take $(\hat{\varphi}, \eta)$ smooth in the definition. But, a posteriori, we see on the resulting normal forms $1.3-1.6$, that such systems are also uniformly infinitesimally identifiable for the (bigger) class of $(\hat{\varphi}, \eta)$ that are $L^{\infty}$ only;

2. second, the following theorem can be proved:

Theorem 5. If $\Sigma$ (analytic) is not infinitesimally identifiable at $\left(x_{0}, \hat{\varphi}\right)$, because of some variation $\eta(\cdot)$ such that $\left(\xi_{0}, \eta\right) \neq 0$ is in the Kernel of $T_{x_{0}, \hat{\varphi}} P_{\Sigma}$, with $(\hat{\varphi}, \eta)$ in $L^{\infty}$, then $\Sigma$ is also not infinitesimally identifiable at some other $\left(x_{1}, \hat{\varphi}_{1}\right)$, because of some other variation $\eta_{1}(\cdot)$ (such that $\left(\xi_{0}^{\prime}, \eta_{1}\right) \neq 0$ is in the Kernel of $\left.T_{x_{1}, \hat{\varphi}_{1}} P_{\Sigma}\right)$, and moreover $\left(\hat{\varphi}_{1}, \eta_{1}\right)$ are analytic.

This theorem shows exactly that (fortunately), the class of $(\hat{\varphi}, \eta)$ in Definition 3 of Uniform Infinitesimal Identifiability, is in fact irrelevant. This result is stronger than the considerations in 1 . above, that hold, as the normal forms, locally around points of an open dense subset of $X \times I$.

We will not give here the proof of this theorem: it is difficult, and far beyond the scope of this paper. It is related to ideas developed in [7] (where comparable results can be found), and also to ideas of "trajectory regularity" that can be found in [17].

\subsection{An example: Biological reactors}

\subsubsection{Presentation}

A simple biological reactor is a process where a population grows in presence of some substrate by eating the substrate. The concentration of the biomass (the population) in the mixture is denoted by $x$, and the concentration of the substrate is $s$. Here, $x$ and $s$ belong to $\mathbb{R}^{+}$. The reactor is fed continuously by some flow of substrate, with concentration $S_{i n}$, and flowrate $D(t)>0 . S_{i n}$ is a constant (in first approximation), and $D(t)$ is usually a control variable.

The way the population grows in presence of substrate is described by a "growth function" $\mu(x, s)$. The total volume is maintained constant by perpetually throwing out the same volume of mixture as the volume of substrate entering the reactor.

Hence, the equations are:

$$
\begin{aligned}
& \frac{\mathrm{d} s}{\mathrm{~d} t}=-\mu \cdot x+D\left(S_{i n}-s\right) \\
& \frac{\mathrm{d} x}{\mathrm{~d} t}=(\mu-D) x .
\end{aligned}
$$


The questions of observation and control of such biological reactor has been treated by several authors. See for instance [4], and the book [2].

In the literature, there are many possible choices for the function $\mu$. One can find a cornucopia of them in the book [2].

Here, we will consider the case where this function $\mu$ is an unknown function, of (either the variable $s$ only, or the variable $x$ only, or of both), and we will give several conclusion about its identifiability.

Remark 6. The biological reactor will provide an example where the identifiability property depends on the internal mapping $\pi$, see Section 7.2. Also, clearly, in that case, identifying the "open-loop" function $\varphi(t)$ is not equivalent to identifying the "closed-loop" function $\varphi \circ \pi(\chi(t))$, with $\chi=(x, s)$.

In fact, the general study in this paper was initially motivated by this very interesting simple example.

\subsubsection{Main theoretical result for the biological reactor}

One of our results for the biological reactor will be like that: we will consider that there is a single observation, the concentration $s$ of the substrate.

Then, the biological reactor is not identifiable in the sense of Definition 1.

Also, if we consider only constant input functions, $(D(\cdot)=c t$.), then, the (single output system is not uniformly infinitesimally identifiable in the sense of Definition 3, because it does not meet the necessary conditions of Theorem 2.

Assuming that the growth function depends on $s$ only $(\pi:(x, s) \rightarrow s$ is the internal mapping), then, we prove (easily) the following theorem:

Theorem 6. The bioreactor is identifiable at an admissible i-o trajectory $(y(\cdot), u(\cdot))=(s(\cdot), D(\cdot)$ iff the output trajectory $y(\cdot)=s(\cdot)$ visits twice the same value.

This result, with others, is proved in Section 7.

\section{THE GENERIC CASE}

The purpose of this section is to prove Theorem 1, and to give some complementary results and examples.

\subsection{Comparison between differential identifiability and identifiability}

We state a result in the uncontrolled case only. In the controlled case, the situation is much more complicated. It is possible to prove something very strong, using techniques developed in the book [7], for observability. But, this is a great complication, and it will be the purpose of another paper.

Theorem 7 (uncontrolled systems). In the uncontrolled case, differential identifiability at some order implies identifiability, whatever the internal mapping $\pi$.

Proof. Let us assume that an uncontrolled system $\Sigma$ is not identifiable. It means that there is an admissible output trajectory $\hat{y}:\left[0, \tau\left[\rightarrow \mathbb{R}^{d_{y}}\right.\right.$, such that $\Sigma$ is not identifiable at $\hat{y}$, i.e., there are two trajectories $x_{1}(t), x_{2}(t)$, and two corresponding functions $\hat{\varphi}_{1}(t), \hat{\varphi}_{2}(t), \hat{\varphi}_{1}(t)=\varphi_{1}\left(\pi\left(x_{1}(t)\right)\right), \hat{\varphi}_{2}(t)=\varphi_{2}\left(\pi\left(x_{2}(t)\right)\right)$, such that $\varphi_{1}$ and $\varphi_{2}$ are smooth, hence $\hat{\varphi}_{1}(t), \hat{\varphi}_{2}(t)$, and $\hat{y}(t)$ are smooth. Moreover, $\hat{\varphi}_{1}\left(t_{0}\right) \neq \hat{\varphi}_{2}\left(t_{0}\right)$ for some $t_{0} \in[0, \tau[$, or $x_{1}(0) \neq x_{2}(0)$. Restricting the interval $\left[0, \tau\right.$ [, we may assume that $t_{0}=0$. Then, for an arbitrary positive integer $k$, consider the two $k$-jets at time zero, $j^{k} \hat{\varphi}_{1}, j^{k} \hat{\varphi}_{2}$. Consider the mapping $\Phi_{k}^{\Sigma}$ defined in the introduction. Set $z_{1}=\left(x_{1}(0), j^{k} \hat{\varphi}_{1}\right), z_{2}=\left(x_{2}(0), j^{k} \hat{\varphi}_{2}\right)$. Then, $\left(\Phi_{k}^{\Sigma}\left(z_{1}\right), \Phi_{k}^{\Sigma}\left(z_{2}\right)\right) \in \Delta_{k}$, the diagonal of $\mathbb{R}^{k d_{y}} \times \mathbb{R}^{k d_{y}}$. Therefore, $\Sigma$ is not differentially identifiable of order $k$, since $\hat{\varphi}_{1}(0) \neq \hat{\varphi}_{2}(0)$ or $x_{1}(0) \neq x_{2}(0)$. 


\subsection{Preliminaries for the proof of Theorem 1}

Let $S$ denote the set of $C^{\infty}$ systems $\Sigma=(f, h)$ of the form 1.2, i.e. elements of $S$ are couples $(f, h)$ of $\varphi$-parametrized vector fields $f$ and functions $h$. We endow $S$ with the $C^{\infty}$ Whitney topology. Let $J^{k} S$ denote the bundle of $k$-jets of systems in $S$. It is the fiber product $J^{k} F \times_{X \times I} J^{k} H$ of the bundles $J^{k} F, J^{k} H$ over $X \times I$, that are respectively the bundles of $k$-jets of smooth sections of $T X \times I \rightarrow X \times I$ and $X \times I \times \mathbb{R}^{d_{y}} \rightarrow X \times I$.

Let us also denote by $J^{k} S_{2}^{*}$ the restriction of $J^{k} S^{2}=J^{k} S \times J^{k} S$ to $((X \times I) \times(X \times I)) \backslash \Delta(X \times I)$, where $\Delta(X \times I)$ is the diagonal in $((X \times I) \times(X \times I))$.

Let us recall that the mappings $\Phi_{k}^{\Sigma}, \Phi_{k, 2}^{\Sigma, *}$, defined in the Introduction, depend only on the $k$-jet $j^{k} \Sigma$ of $\Sigma$. When we want to stress this fact, we write $\Phi^{j^{k} \Sigma}$ in place of $\Phi_{k}^{\Sigma}$.

3.2.1. The bad sets

Definition 4. $B_{1}(k)$ is the subset of $J^{k} S^{2}$ of all couples $\left(j^{k} \Sigma(p), j^{k} \Sigma(q)\right)$, such that: (1) $p \neq q, p=\left(x_{1}, \varphi_{1}\right)$, $q=\left(x_{2}, \varphi_{2}\right),(2) f(p)=f(q)=0,(3) h(p)=h(q)$.

Definition 5. a. Let $\hat{B}_{2}(k)$ be the subset of $J^{k} S^{2} \times \mathbb{R}^{(k-1)} \times \mathbb{R}^{(k-1)}$, of all tuples $\left(j^{k} \Sigma(p), j^{k} \Sigma(q), v_{1}, v_{2}\right)$ such that: (1) $p \neq q, p=\left(x_{1}, \varphi_{1}\right), q=\left(x_{2}, \varphi_{2}\right),(2) f(p) \neq 0$ or $f(q) \neq 0,(3) \Phi_{k}^{\Sigma}\left(x_{1},\left(\varphi_{1}, v_{1}\right)\right)=\Phi_{k}^{\Sigma}\left(x_{2},\left(\varphi_{2}, v_{2}\right)\right)$,

b. $B_{2}(k)$ denotes the canonical projection of $\hat{B}_{2}(k)$ in $J^{k} S^{2}$.

The two following lemmas are obvious.

Lemma 1. $B_{1}(k), \hat{B}_{2}(k), B_{2}(k)$, are respectively partially semi-algebraic subbundles of $J^{k} S_{2}^{*}, J^{k} S_{2}^{*} \times \mathbb{R}^{(k-1)} \times$ $\mathbb{R}^{(k-1)}, J^{k} S_{2}^{*}$ (this means that heir typical fiber is a semi-algebraic subset of the fibers of the ambient bundles).

Lemma 2. If the map

$$
\begin{gathered}
\Theta:((X \times I) \times(X \times I)) \backslash \Delta(X \times I) \rightarrow j^{k} S_{2}^{*} \\
(p, q) \rightarrow\left(j^{k} \Sigma(p), j^{k} \Sigma(q)\right), p=\left(x_{1}, \varphi_{1}\right), q=\left(x_{2}, \varphi_{2}\right),
\end{gathered}
$$

avoids $B_{1}(k) \cup B_{2}(k)$, then $\Sigma$ is differentially identifiable of order $k$.

\subsection{Proof of Theorem 1}

a. Estimation of the codimension of $B_{1}(k)$ in $J^{k} S_{2}^{*}$ : It is obvious that this codimension is $2 n+d_{y}$.

b. Estimation of the codimension of $B_{2}(k)$ in $J^{k} S_{2}^{*}$ : We treat only the case $f(p) \neq 0$. The other case $f(q) \neq 0$ is similar.

Let $(x, \varphi, y, \psi) \in((X \times I) \times(X \times I)) \backslash \Delta(X \times I)$. The typical fiber $\hat{B}_{2}(k, x, \varphi, y, \psi)$ of $\hat{B}_{2}(k)$ in $J^{k} S(x, \varphi) \times$ $J^{k} S(y, \psi) \times \mathbb{R}^{k-1} \times \mathbb{R}^{k-1}$ is characterized by the following properties: (i) $f(p) \neq 0$ and $(i i) \Phi_{k}^{\Sigma}\left(x,\left(\varphi, v_{1}\right)\right)-\Phi_{k}^{\Sigma}(y$, $\left.\left(\psi, v_{2}\right)\right)=0$.

Let $G$ be the subset of $J^{k} S(x, \varphi) \times J^{k} S(y, \psi) \times \mathbb{R}^{k-1} \times \mathbb{R}^{k-1}$ of all tuples $\left(j^{k} \Sigma(x, \varphi), v_{1}, j^{k} \Sigma(y, \psi), v_{2}\right)$ such that $f(x, \varphi) \neq 0$ and let $\chi: G \rightarrow \mathbb{R}^{k}$ be the mapping $\chi\left(j^{k} \Sigma(x, \varphi), v_{1}, j^{k} \Sigma(y, \psi), v_{2}\right)=\Phi_{k}^{\Sigma}\left(x,\left(\varphi, v_{1}\right)\right)-\Phi_{k}^{\Sigma}(y$, $\left.\left(\psi, v_{2}\right)\right)$. Then, $\hat{B}_{2}(k, x, \varphi, y, \psi)=\chi^{-1}(0)$.

The map $\chi$ is an algebraic mapping, affine in $j^{k} h(x, \varphi)$.

By Lemma 3 in Appendix 3.5 below, for fixed $v_{1} \in \mathbb{R}^{k}$ and $j^{k} f(x, \varphi)$, the linear mapping $j^{k} h(x, \varphi) \in$ $J^{k} H(x, \varphi) \rightarrow \Phi^{j^{k} \Sigma}\left(x, \varphi, v_{1}\right)$ is surjective. This shows that the map $\chi: G \rightarrow \mathbb{R}^{k}$ is a submersion. Since $\hat{B}_{2}(k, x, \varphi, y, \psi)=\chi^{-1}(0)$,

$$
\operatorname{codim}\left(\hat{B}_{2}(k, x, \varphi, y, \psi), J^{k} S(x, \varphi) \times J^{k} S(y, \psi) \times \mathbb{R}^{k-1} \times \mathbb{R}^{k-1}\right)=k d_{y} .
$$

Hence:

$$
\operatorname{codim}\left(\hat{B}_{2}(k), J^{k} S_{2}^{*} \times \mathbb{R}^{k-1} \times \mathbb{R}^{k-1}\right)=k d_{y}
$$


It follows that $\operatorname{codim}\left(B_{2}(k), J^{k} S_{2}^{*}\right) \geq k\left(d_{y}-2\right)+2$.

c. Final estimation of $\operatorname{codim}\left(B_{1}(k) \cup B_{2}(k), J^{k} S_{2}^{*}\right)$.

It follows from a. and b. above that:

$$
\operatorname{codim}\left(B_{1}(k) \cup B_{2}(k), J^{k} S_{2}^{*}\right) \geq \min \left(2 n+d_{y}, k\left(d_{y}-2\right)+2\right) .
$$

d. Proof of Theorem 1. Let $k \geq 2 n+1$, and $d_{y} \geq 3$. Then, $\operatorname{codim}\left(B_{1}(k) \cup B_{2}(k), J^{k} S_{2}^{*}\right) \geq 2 n+3$. We apply the standard multijet transversality theorems ( [1], [11]) to the map

$$
\begin{gathered}
\rho: S \times((X \times I) \times(X \times I)) \backslash \Delta(X \times I) \rightarrow J^{k} S_{2}^{*}, \\
(\Sigma, x, \varphi, y, \psi) \rightarrow\left(j^{k} \Sigma(x, \varphi), j^{k} \Sigma(y, \psi)\right) .
\end{gathered}
$$

This allows to conclude that the set of $\Sigma \in S$ such that $\rho_{\Sigma}$ is transverse to $B_{1}(k) \cup B_{2}(k)$ is residual. But, $\operatorname{dim}(((X \times I) \times(X \times I)) \backslash \Delta(X \times I))=2 n+2<\operatorname{codim}\left(B_{1}(k) \cup B_{2}(k), J^{k} S_{2}^{*}\right) \geq 2 n+3$.

Hence, the set of $\Sigma \in S$ such that $\rho_{\Sigma}$ avoids $B_{1}(k) \cup B_{2}(k)$ is residual. By Lemma 2, this ends the proof of Theorem 1.

\subsection{A counterexample}

We consider, on the circle $S^{1}$, for values of $\varphi$ in the interval $I=[-\pi, \pi]$, systems of the form: $\Sigma=\Sigma_{0}+\delta \Sigma$, where $\delta \Sigma$ is $C^{\infty}$ but $C^{1}$ small, and $\Sigma_{0}$ is the system:

$$
\Sigma_{0}\left\{\begin{array}{l}
\dot{x}=\psi(x)-\varphi=f_{0}(x, \varphi) \\
y_{1}=\cos (x)=h_{0}^{1}(x, \varphi) \\
y_{2}=\sin (2 x)=h_{0}^{2}(x, \varphi) .
\end{array}\right.
$$

The cyclic coordinate on $S^{1}$ is $x$ and $\psi: S^{1} \rightarrow \mathbb{R}$ is such that $\psi(x)=x$ on the interval $\left[-\frac{\pi}{2}-\varepsilon, \frac{\pi}{2}+\varepsilon\right]$, for $\varepsilon>0$ small. We want to solve the system of equations:

$$
\begin{aligned}
\left(f_{0}+\delta f\right)\left(x_{1}, \varphi_{1}\right) & =0 \\
\left(f_{0}+\delta f\right)\left(x_{2}, \varphi_{2}\right) & =0 \\
\left(h_{1}+\delta h_{1}\right)\left(x_{1}, \varphi_{1}\right)-\left(h_{1}+\delta h_{1}\right)\left(x_{2}, \varphi_{2}\right) & =0 \\
\left(h_{2}+\delta h_{2}\right)\left(x_{1}, \varphi_{1}\right)-\left(h_{2}+\delta h_{2}\right)\left(x_{2}, \varphi_{2}\right) & =0
\end{aligned}
$$

around the trivial solution $\delta f=0, \delta h_{1}=0, \delta h_{2}=0, x_{1}=\frac{\pi}{2}, \varphi_{1}=\frac{\pi}{2}, x_{2}=-\frac{\pi}{2}, \varphi_{2}=-\frac{\pi}{2}$.

Here, the set $S$ of systems is identified with a subspace of the Banach space $B S$ of $C^{1}$ triples $\left(\delta f, \delta h_{1}, \delta h_{2}\right) \in$ $\left(C^{1}\left(S^{1} \times I\right)\right)^{3}$ with the $C^{1}$ topology .

It is trivial to check that the Jacobian matrix of the system (3.2), with respect to the variables $\left(x_{1}, \varphi_{1}, x_{2}, \varphi_{2}\right)$ at the point $\left(\frac{\pi}{2}, \frac{\pi}{2},-\frac{\pi}{2},-\frac{\pi}{2}\right)$, is invertible. This means, applying the implicit function theorem in the Banach manifold $B S \times S^{1} \times I \times S^{1} \times I$, that for all $C^{\infty}$ variations $\delta \Sigma$ small enough $\left(C^{1}\right)$, we can find solutions to equations (3.2), and these solutions are close to $x_{1}=\frac{\pi}{2}, \varphi_{1}=\frac{\pi}{2}, x_{2}=-\frac{\pi}{2}, \varphi_{2}=-\frac{\pi}{2}$.

But, such a solution of (3.2) produces a system $\Sigma$ and two couples $\left(x_{1}, \varphi_{1}\right) \neq\left(x_{2}, \varphi_{2}\right)$ such that, for the constant control functions $\varphi_{1}, \varphi_{2}$, and for the initial conditions $x_{1}, x_{2}$, the corresponding couples of outputs $\left(y_{1}, y_{2}\right)$ are constant functions of the time, that are equal.

Hence, there is an open neighborhood $\left(C^{1}\right.$ open in $\left.C^{\infty}\right)$ of systems, that are not differentially identifiable at any order $k$ (and also not identifiable).

Remark 7. Notice that, in the formula (3.1), the 2 bounds on the codimension are $2 n+2$ and 2 , for $d_{y}=2$. Hence, there should be two typologies of counterexamples for $d_{y}=2$. 
In this example, we have exhibited the simplest one, corresponding to codimension $2 n+2$. But, from the results of Section 5 (the case $d_{y}=2$ ), counterexamples corresponding to the other typology are easy to construct.

\subsection{Appendix}

For the sake of completeness, we give here a (very simple) lemma which is already contained in the paper [6], and in the book [7].

If $V$ is a vector space, $\operatorname{Sym}^{a}(V)$ denotes the space of symmetric tensors of degree $a$ on $V$, that can be canonically identified with the space of homogeneous polynomials of degree $a$ over $V^{*}$, dual space of $V$. The symbol $\odot$ means symmetric tensor product or power.

Let $(x, \varphi, v) \in X \times I \times \mathbb{R}^{k-1}$ be given, and set $p=(x, \varphi)$. Let $f \in F$ (the space of smooth $I$-parametrized vector fields on $X$ ), such that $f(p) \neq 0$.

Lemma 3. The mapping $\Theta: J^{k} H \rightarrow \mathbb{R}^{k d_{y}}, j^{k} h \rightarrow \Phi^{j^{k} \Sigma}(x, \varphi, v)$ is linear and surjective.

Proof. Let $f$ be a representative of $j^{k} f(p)$. Take a coordinate system $\left(O, x_{1}, \ldots, x_{n}\right)$ for $X$ at $x$. Then, if $y^{(r)}$ denotes the $r^{\text {th }}$ derivative of the output at time 0 , we have:

$$
y^{(r)}(p, v)=d_{x}^{r} h\left(p ; f(p)^{\odot r}\right)+\sum_{a=0}^{r-1} d_{x}^{a} h\left(p, R_{a, r}\left(j^{k} f(p), v\right)+\sum_{\substack{l=1, \ldots, r, s+l=r}} d_{x}^{s} d_{\varphi}^{l} h\left(p ; T_{s, l}^{r}\left(j^{k} f(p), v\right)\right) .\right.
$$

The expressions $R_{a, r}, T_{s, l}^{r}$, are universal polynomial mappings : $J^{k} F(p) \times \mathbb{R}^{k-1} \rightarrow \operatorname{Sym}^{a}\left(T_{x} X\right)\left(\right.$ resp. $J^{k} F(p) \times$ $\left.R^{k-1} \rightarrow \operatorname{Sym}^{s}\left(T_{x} X\right) \otimes \mathbb{R}^{l}\right)$. This implies the result because $f(p) \neq 0$.

\section{The Single-OUtPut CASE}

The purpose of this section is to prove Theorem 2 and Theorem 3.

\subsection{Proof of Theorem 2}

Let us assume (A) that $\frac{\partial}{\partial \varphi}\left(L_{f_{\varphi}}\right)^{i} h_{\varphi} \equiv 0, i=0, \ldots, k-1$, and $\frac{\partial}{\partial \varphi}\left(L_{f_{\varphi}}\right)^{k} h_{\varphi} \neq 0$, for $k \leq n-1$.

Then (B): the closed analytic subset of $X: Z_{k-1}=\left\{x \mid d_{x} h \wedge \ldots \wedge d_{x} L_{f}^{k-1} h(x)=0\right\}$, has codimension 1 at least. Then, for all $x_{0} \in X \backslash Z_{k-1}$, it can be completed by functions $h_{k}(x), \ldots, h_{n}(x)$, in order to form a local coordinate system on a neighborhood $U_{x_{0}}$ of $x_{0}$.

Were it otherwise, then, by connectedness, $d_{x} h \wedge d_{x} L_{f} h \wedge \ldots \wedge d_{x}\left(L_{f}\right)^{k-1} h \equiv 0$. Take $j \leq k-1$, the first integer such that $d_{x} h \wedge d_{x} L_{f} h \wedge \ldots \wedge d_{x}\left(L_{f}\right)^{j} h \equiv 0$. Then, set, on some open subset of $X: x_{1}=h, \ldots, x_{j}=\left(L_{f}\right)^{j-1} h$. Complete this set of functions by $x_{j+1}, \ldots, x_{n}$, in order to form a coordinate system.

In these coordinates, with a straightforward computation, the system $\Sigma$ can be rewritten:

$$
\sum\left\{\begin{array}{c}
y=x_{1}, \\
\dot{x}_{1}=x_{2}, \\
\cdot \\
\cdot \\
\dot{x}_{j-1}=x_{j}, \\
\dot{x}_{j}=\psi_{j}\left(x_{1}, \ldots, x_{j}\right), \\
\dot{x}_{j+1}=\psi_{j+1}(x, \varphi) \\
\dot{x_{n}}=\dot{\psi}_{n}(x, \varphi),
\end{array}\right.
$$


which is obviously not uniformly infinitesimally identifiable: take in the first variation, $\xi(0) \neq 0, \xi_{1}(0)=$ $\cdots=\xi_{j}(0)=0$, and the "variation" $\eta(\cdot) \equiv 0$. The output of the first variation system is identically zero, whatever $\varphi(\cdot)$. A contradiction with uniform infinitesimal identifiability.

This shows (B).

Now, let us show that (A) is impossible.

In the coordinate system defined as in (B), the system writes, on some open subset:

$$
\sum\left\{\begin{array}{c}
y=x_{1}, \\
\dot{x}_{1}=x_{2}, \\
\cdot \\
\cdot \\
\dot{x}_{k-1}=x_{k}, \\
\dot{x}_{k}=\psi_{k}(x, \varphi), \\
\cdot \\
\cdot \\
\dot{x}_{n}=\psi_{n}(x, \varphi) .
\end{array}\right.
$$

Now, the linearized system writes, in these coordinates (and the associated coordinates $\xi_{i}$ on $T X$ ):

$$
\left\{\begin{array}{c}
\dot{x}=f(x, \varphi) \\
\dot{\xi}_{1}=\xi_{2} \\
\dot{\xi}_{k-1}=\xi_{k} \\
\dot{\xi}_{i}=d_{x} \psi_{i}(x, \varphi) \xi+d_{\varphi} \psi_{i}(x, \varphi) \eta \\
i=k, \ldots, n
\end{array}\right.
$$

where $f$ is as in (4.1). Let us take $\xi(0) \neq 0, \xi_{1}(0)=0, \ldots, \xi_{k}(0)=0$. Our assumption (A) implies that on an open dense semianalytic subset $D$ of $X \times I, d_{\varphi} \psi_{k}(x, \varphi) \neq 0$. Around a point $p_{0}=\left(x_{0}, \xi(0), \varphi_{0}\right) \in T X \times I,\left(x_{0}, \varphi_{0}\right) \in D$, with $\xi(0)$ just defined, we consider the feedback function for the system 4.2: $\eta(x, \varphi, \xi)=\frac{-\mathrm{d}_{x} \psi_{k}(x, \varphi) \xi}{\mathrm{d}_{\varphi} \psi_{k}(x, \varphi)}$. We consider also the function $\varphi(t) \equiv \varphi_{0}$. Then, the feedback system (4.2) has a unique trajectory $(\xi(t), x(t))$ around $p_{0}$, starting from $\left(x_{0}, \xi_{0}\right)$ at time 0 . By construction, this trajectory is such that $\xi_{1}(t), \ldots, \xi_{k}(t)=0$ for all $t$ small enough. This contradicts the infinitesimal identifiability assumption. Hence, $\frac{\partial}{\partial \varphi}\left(L_{f_{\varphi}}\right)^{k} h_{\varphi}=0,(\mathrm{~A})$ is impossible.

Now, at this point, identically on $X \times I, \frac{\partial}{\partial \varphi}\left\{\left(L_{f_{\varphi}}\right)^{k} h_{\varphi}=0\right.$, for $k=0, \ldots, n-1$. This is the property 1.a. of Theorem 2. Property 1.c. also holds, by the proof of (B) above, which works also in that case. Moreover, we already know that, on an open dense semianalytic subset $D$ of $X$, our system can be rewritten (in local coordinates, around any point $\left.x_{0} \in D\right)$ :

$$
\left\{\begin{array}{c}
y=x_{1}, \\
\dot{x}_{1}=x_{2}, \\
\cdot \\
\cdot \\
\dot{x}_{n-1}=x_{n}, \\
\dot{x}_{n}=\psi(x, \varphi) .
\end{array}\right.
$$

Consider the closed analytic subset $S \subset X \times I$, formed by the $(x, \varphi)$ 's satisfying $\frac{\partial \psi}{\partial \varphi}=0$ (or equivalently, $\frac{\partial}{\partial \varphi}\left\{\left(L_{f_{\varphi}}\right)^{n} h_{\varphi}=0\right)$. If this subset has nonempty interior, by analyticity and connectedness, it is the whole $X \times I$, and $\psi$ does not depend on $\varphi$, which is easily seen as a contradiction with infinitesimal identifiability. Then, $S$ has codimension 1 at least. Let $\Pi S$ be the projection of $S$ on $X, \Pi: X \times I \rightarrow X$. Since $I$ is compact, $\Pi S$ is subanalytic. By Hardt's theorem [9], we can stratify the mapping $\Pi: S \rightarrow \Pi S$. Let $S_{1}$, $\Pi S_{1}$ be two strata such that $\Pi S_{1}$ has maximal dimension, and $\Pi$ maps $S_{1}$ submersively onto $\Pi S_{1}$. Let $\hat{\varphi}: \Pi S_{1} \rightarrow S_{1}$ be a smooth section of $\Pi$. (See the footnote at the beginning of the Appendix, Sect. A). 
Assume that $\operatorname{dim}\left(\Pi S_{1}\right)=\operatorname{dim} X=n$. Then, $\hat{\varphi}$ is a smooth mapping from an open subset $\Pi S_{1}$ of $X$ to $X \times I$.

Let us apply this (feedback) function $\hat{\varphi}$ to our linearized system restricted to $\Pi S_{1} \times I$. In the coordinates defined above, it rewrites:

$$
\begin{aligned}
y & =x_{1} \\
\dot{x}_{1} & =x_{2}, \ldots, \dot{x}_{n-1}=x_{n}, \dot{x}_{n}=\psi(x, \hat{\varphi}(x)), \\
\dot{\xi}_{1} & =\xi_{2}, \ldots, \dot{\xi}_{n-1}=\xi_{n}, \dot{\xi}_{n}=d_{x} \psi(x, \hat{\varphi}(x)) \xi+0 . \\
\hat{y} & =\xi_{1} .
\end{aligned}
$$

This equation (4.4) is independent of $\eta$, the input of the linearized system. Then, let us take $\xi(0)=0$, $\eta(t) \neq 0$. Then $\hat{y}(t)$ is identically zero, which contradicts the infinitesimal identifiability.

Therefore, $\Pi S_{1}$ is not open in $X$, and therefore, it has codimension 1 . $\Pi S$ has codimension 1 , and this shows exactly the property 1.b. of Theorem 2, together with the property 2 . (the normal form), in the statement of the theorem. This ends the proof.

\subsection{Proof of Theorem 3}

Assume that $\Sigma$ is a system in normal form (4.3), on some open neighborhood $O$ of $x=0$ in $\mathbb{R}^{n}$, with $\frac{\partial \psi}{\partial \varphi}(x)$ never vanishing.

It is clear that admissible output trajectories (there is no input in our case), are smooth. Given any smooth function $y(\cdot):\left[0, T\left[\rightarrow \mathbb{R}\right.\right.$, an immediate computation with the normal form (4.3) shows that $x_{1}(t)=y(t)$, $x_{2}(t)=\dot{y}(t), \ldots, x_{n}(t)=\frac{\mathrm{d}^{n-1} y}{\mathrm{~d} t^{n-1}}(t)$. Also,

$$
\frac{\mathrm{d}^{n} y}{\mathrm{~d} t^{n}}(t)=\psi\left(y, \dot{y}, \ldots, y^{(n-1)}(t), \varphi(t)\right)
$$

Assume that $y(\cdot)$ is an admissible output trajectory. For $y, \dot{y}, \ldots, y^{(n-1)}$ fixed, set:

$$
\psi_{y, \dot{y}, \ldots, y^{(n-1)}}(\varphi)=\psi\left(y, \dot{y}, \ldots, y^{(n-1)}, \varphi\right)
$$

The function $\psi_{y, \dot{y}, \ldots, y^{(n-1)}}$ is monotonous: $\frac{\partial \psi}{\partial \varphi}(\cdot)$ never vanishes. Since $y(\cdot)$ is an admissible output trajectory, then, the equation (4.5) has a solution $\varphi(t)$ for all $t \in[0, T[$ (and this solution is smooth w.r.t. $t$ ). By the monotonicity, this solution is unique. This means that $\Sigma$ is identifiable, for $Z=O$ and $\pi: O \rightarrow O$ being the trivial "internal mapping". For the same reason, it is also identifiable if $\pi: O \rightarrow Z$, is nontrivial.

To finish, by the normal form, it is just a matter of trivial computation to show that $\Sigma_{\mid O}$ is differentially identifiable of order $n+1$, and the uniform infinitesimal identifiability of $\Sigma_{\mid O}$ is also obvious, from the normal form.

\section{The TWO-OUtPut CASE}

Here, we want to prove Theorem 4, give a series of intrinsic conditions corresponding to these normal forms, state and prove several weak converses of Theorem 4 for all these normal forms, and give a few examples.

\subsection{Preliminaries, definitions}

Here, as above, $L$ is the Lie derivative operator on $X$. Hence, if $f(x, \varphi)$ is a $\varphi$-dependant vector field, $L_{f}$ is the Lie derivative operator with respect to the vector fields $f_{\varphi}(x)=f(x, \varphi)$, for $\varphi$ fixed in $I$. 
Let $N(l)$ be the rank at generic points of $X \times I$ of the family $E_{l}$ of one-forms on $X$ :

$$
E_{l}=\left\{d_{x} h_{i}, d_{x} L_{f} h_{i}, \ldots, d_{x} L_{f}^{l-1} h_{i}, i=1,2\right\}
$$

Set $N(0)=0$.

This set of generic points $U_{l}$, is the intersection of the open sets $\tilde{U}_{i}, i \leq l$, where $E_{i}$ has maximal rank. $U_{l}$ is semianalytic, open and dense in $X \times I$. Moreover, $U_{l+1} \subset U_{l}$.

It is easy to check that $N(l)$ increases strictly by steps of 2 , up to $l=k$, and after, (eventually), it increases by steps of 1 up to $l=l_{M}, N\left(l_{M}\right) \leq n$.

It may happen that $k=0$, i.e. $N(1)=1$.

Lemma 4. If $\Sigma$ is uniformly infinitesimally identifiable, then, $N\left(l_{M}\right)=n$.

The idea in this lemma is that, if it is not true, then, for constant functions $\varphi(\cdot)$, infinitesimal identifiability will be contradicted.

Proof. If $N\left(l_{M}\right)<n$, let $\left(x_{1}, \ldots, x_{n}, \varphi\right)$ be a coordinate system in an open subset of $U_{l_{M}}$ formed by $\varphi$, and by $N\left(l_{M}\right)$ functions of $(x, \varphi)$ chosen, in the family $\left\{h_{i}, L_{f} h_{i}, \ldots, L_{f}^{l_{M}-1} h_{i}, i=1,2\right\}$, (the $N\left(l_{M}\right)$ first coordinates), and other $x$-coordinates. In these coordinates, it is easy to see that, for the constant function $\varphi(\cdot) \equiv \varphi_{0}, \Sigma$ can be rewritten as:

$$
\begin{gathered}
\dot{x}_{1}=f_{1}\left(x_{1}, \ldots, x_{N\left(l_{M}\right)}, \varphi_{0}\right), \\
\cdot \cdot \\
\dot{x}_{N\left(l_{M}\right)}=f_{N\left(l_{M}\right)}\left(x_{1}, \ldots, x_{N\left(l_{M}\right)}, \varphi_{0}\right), \\
\dot{x}_{N\left(l_{M}\right)+1}=f_{N\left(l_{M}\right)+1}\left(x_{1}, \ldots, x_{n}, \varphi_{0}\right), \\
\cdot \\
\dot{x}_{n}=f_{n}\left(x_{1}, \ldots, x_{n}, \varphi_{0}\right), \\
y_{1}=h_{1}\left(x_{1}, \ldots, x_{N\left(l_{M}\right)}, \varphi_{0}\right), \\
y_{2}=h_{2}\left(x_{1}, \ldots, x_{N\left(l_{M}\right)}, \varphi_{0}\right) .
\end{gathered}
$$

Then, taking $\eta \equiv 0$, and $\xi_{1}(0)=\cdots=\xi_{N\left(l_{M}\right)}(0)=0, \xi_{N\left(l_{M}\right)+1}(0) \neq 0$, in the equation of the first variation, we see that the solution $\xi(t)$ verifies $\xi_{1}(t)=\cdots=\xi_{N\left(l_{M}\right)}(t)=0$, on a small time interval $[0, T], T>0$. This implies that on this time interval, the output $\hat{y}=\left(\hat{y}_{1}, \hat{y}_{2}\right)$ of the first variation is identically zero. A contradiction with infinitesimal identifiability for $\varphi(\cdot) \equiv \varphi_{0}$.

Definition 6. We define $r$, the "order" of the system, as the first integer such that $d_{\varphi} L_{f}^{r} h$ does not vanish identically.

Lemma 5. If $\Sigma$ is uniformly infinitesimally identifiable, then, $r \leq l_{M}$.

Proof. Assume $r \geq l_{M}+1$. Let us take again a coordinate system on an open subset of $X$, formed by functions of the family $\left\{h_{i}, L_{f} h_{i}, \ldots, L_{f}^{l_{M}-1} h_{i}, i=1,2\right\}$. These functions are functions of $x$ only, since $r \geq l_{M}+1$. By the previous lemma, this is possible. It is obvious that, in these coordinates, the system can be rewritten:

$$
\dot{x}=f(x), \quad y=h(x) .
$$

Let us take $\xi(0)=0$, but a function $\eta(t)$ nonzero, in the first variation. Then, the output $\hat{y}$ of the first variation is identically zero, on some open time interval. This contradicts the infinitesimal identifiability.

Definition 7. A system $\Sigma$ is regular if $N\left(l_{M}\right)=n$ and $r \leq l_{M}$. 
If a system is uniformly infinitesimally identifiable, then it is regular, by the 2 previous lemmas. From now on, in this section, we will assume that systems $\Sigma$ under consideration are regular.

The integer $k$ is the first with the following properties:

$$
\begin{gathered}
d_{x} h_{1} \wedge d_{x} h_{2} \wedge d_{x} L_{f} h_{1} \wedge \ldots \wedge d_{x} L_{f}^{k} h_{1} \wedge d_{x} L_{f}^{k} h_{2} \equiv 0, \text { but } \\
d_{x} h_{1} \wedge d_{x} h_{2} \wedge d_{x} L_{f} h_{1} \wedge \ldots \wedge d_{x} L_{f}^{k-1} h_{1} \wedge d_{x} L_{f}^{k-1} h_{2} \neq 0 \text { (not identically zero) } .
\end{gathered}
$$

If $r=k$, there are three possibilities:
A. $n=2 k$;
B.

B.1.

$$
d_{x} h_{1} \wedge d_{x} h_{2} \wedge d_{x} L_{f} h_{1} \wedge \ldots \wedge d_{x} L_{f}^{k-1} h_{2} \wedge d_{x} L_{f}^{k} h_{1} \neq 0
$$

(hence $n>2 k$ ) and $d_{\varphi} L_{f}^{k} h_{2} \neq 0$; or,

B.2.

(hence $n>2 k$ ) and $d_{\varphi} L_{f}^{k} h_{1} \neq 0$;

$$
d_{x} h_{1} \wedge d_{x} h_{2} \wedge d_{x} L_{f} h_{1} \wedge \ldots \wedge d_{x} L_{f}^{k-1} h_{2} \wedge d_{x} L_{f}^{k} h_{2} \neq 0
$$

C.

C.1

$$
d_{x} h_{1} \wedge d_{x} h_{2} \wedge d_{x} L_{f} h_{1} \wedge \ldots \wedge d_{x} L_{f}^{k-1} h_{2} \wedge d_{x} L_{f}^{k} h_{1} \neq 0
$$

(hence $n>2 k$ ) and $d_{\varphi} L_{f}^{k} h_{2} \equiv 0, d_{x} h_{1} \wedge d_{x} h_{2} \wedge d_{x} L_{f} h_{1} \wedge \ldots \wedge d_{x} L_{f}^{k-1} h_{2} \wedge d_{x} L_{f}^{k} h_{2} \equiv 0$,

or

C.2

$$
d_{x} h_{1} \wedge d_{x} h_{2} \wedge d_{x} L_{f} h_{1} \wedge \ldots \wedge d_{x} L_{f}^{k-1} h_{2} \wedge d_{x} L_{f}^{k} h_{2} \neq 0
$$

(hence $n>2 k$ ) and $d_{\varphi} L_{f}^{k} h_{1} \equiv 0, d_{x} h_{1} \wedge d_{x} h_{2} \wedge d_{x} L_{f} h_{1} \wedge \ldots \wedge d_{x} L_{f}^{k-1} h_{2} \wedge d_{x} L_{f}^{k} h_{1} \equiv 0$

Definition 8. Let $\Sigma$ be a regular system. We say that $\Sigma$ has:

- type 1 if $r>k$, or $r=k$ but $\mathbf{C}$. is satisfied;

- type 2 if $r<k$, or $r=k$ but B. is satisfied;

- type 3 if $r=k$ and A. is satisfied.

Lemma 6. Types 1, 2 and 3 exhaust the class of regular systems, and form a partition of this class.

Proof. For a system with $r \neq k$, it is clear that it is either of type 1 or type 2 and not both. There can be only problems for $r=k$. If we show that, for $r=k$ :

i) cases B. and C. exhaust all regular systems with $n>2 k$;

ii) cases B. and C. do not intersect;

then, the theorem is proved since $n \geq 2 k$.

Assume that $\Sigma$ is simultaneously $B$. and $C$., then:

If $\Sigma$ is B.1., it cannot be C.2. which contradicts $d_{x} h_{1} \wedge d_{x} h_{2} \wedge d_{x} L_{f} h_{1} \wedge \ldots \wedge d_{x} L_{f}^{k-1} h_{2} \wedge d_{x} L_{f}^{k} h_{1} \neq 0$, and it cannot be C.1., which contradicts $d_{\varphi} L_{f}^{k} h_{2} \neq 0$.

On the same way, if $\Sigma$ is B.2., it cannot be C.1. which contradicts $d_{x} h_{1} \wedge d_{x} h_{2} \wedge d_{x} L_{f} h_{1} \wedge \ldots \wedge d_{x} L_{f}^{k-1} h_{2} \wedge$ $d_{x} L_{f}^{k} h_{2} \neq 0$, and it cannot be C.2., which contradicts $d_{\varphi} L_{f}^{k} h_{1} \neq 0$.

This shows ii).

Proof of i): By definition of $k$ :

$$
\begin{array}{r}
d_{x} h_{1} \wedge d_{x} h_{2} \wedge d_{x} L_{f} h_{1} \wedge \ldots \wedge d_{x} L_{f}^{k} h_{1} \wedge d_{x} L_{f}^{k} h_{2} \equiv 0 \\
d_{x} h_{1} \wedge d_{x} h_{2} \wedge d_{x} L_{f} h_{1} \wedge \ldots \wedge d_{x} L_{f}^{k-1} h_{1} \wedge d_{x} L_{f}^{k-1} h_{2} \neq 0 .
\end{array}
$$


Since $n>2 k$, either:

(a) $d_{x} h_{1} \wedge d_{x} h_{2} \wedge d_{x} L_{f} h_{1} \wedge \ldots \wedge d_{x} L_{f}^{k-1} h_{1} \wedge d_{x} L_{f}^{k-1} h_{2} \wedge d_{x} L_{f}^{k} h_{1} \neq 0$

or:

$$
\text { (b) } d_{x} h_{1} \wedge d_{x} h_{2} \wedge d_{x} L_{f} h_{1} \wedge \ldots \wedge d_{x} L_{f}^{k-1} h_{1} \wedge d_{x} L_{f}^{k-1} h_{2} \wedge d_{x} L_{f}^{k} h_{2} \neq 0
$$

or both: were it otherwise, $l_{M}=k, N\left(l_{M}\right)=2 k$, and $n=2 k$, by Lemma 4 .

Assume that (a) and (b) hold simultaneously.

Then, since $d_{\varphi} L_{f}^{k} h=d_{\varphi} L_{f}^{r} h \neq 0$ by definition of $r$, either $(\alpha), d_{\varphi} L_{f}^{k} h_{1} \neq 0$, or $(\beta), d_{\varphi} L_{f}^{k} h_{2} \neq 0$ Assume $(\alpha)$ (the case $(\beta)$ is symmetric). Then we are in case B.2.

Assume that $(a)$ holds, but not $(b)$ (the case $(b)$ holds but not $(a)$ is symmetric). Then:

$$
\begin{aligned}
& d_{x} h_{1} \wedge d_{x} h_{2} \wedge d_{x} L_{f} h_{1} \wedge \ldots \wedge d_{x} L_{f}^{k-1} h_{1} \wedge d_{x} L_{f}^{k-1} h_{2} \wedge d_{x} L_{f}^{k} h_{1} \neq 0, \\
& d_{x} h_{1} \wedge d_{x} h_{2} \wedge d_{x} L_{f} h_{1} \wedge \ldots \wedge d_{x} L_{f}^{k-1} h_{1} \wedge d_{x} L_{f}^{k-1} h_{2} \wedge d_{x} L_{f}^{k} h_{2} \equiv 0 .
\end{aligned}
$$

If $d_{\varphi} L_{f}^{k} h_{2} \neq 0$, we are in case B.1., if $d_{\varphi} L_{f}^{k} h_{2} \equiv 0$, we are in case C.1. This ends the proof.

Type 2 regular systems. For a regular system of type 2 , eventually interchanging the role of $h_{1}$, $h_{2}$, we can assume that $d_{\varphi} L_{f}^{r} h_{2}(x, \varphi) \neq 0$. In a neighborhood of a point $\left(x_{0}, \varphi_{0}\right) \in U_{l_{M}}$, such that $L_{f}^{r} h_{2}\left(x_{0}, \varphi_{0}\right)=u_{0}$ and $d \varphi L_{f}^{r} h_{2}\left(x_{0}, \varphi_{0}\right) \neq 0$, there is an analytic function $\Phi^{*}(x, u)$, such that $L_{f}^{r} h_{2}\left(x, \Phi^{*}(x, u)\right)=u$. Let us consider the "auxiliary system" $\Sigma_{A}$ :

$$
\Sigma_{A}\left\{\begin{array}{l}
\dot{x}=f\left(x, \Phi^{*}(x, \tilde{\varphi})\right)=F(x, \tilde{\varphi}) \\
y=h\left(x, \Phi^{*}(x, \tilde{\varphi})\right)=H(x, \tilde{\varphi}) .
\end{array}\right.
$$

This system is well defined and intrinsic, over an open set $V_{x_{0}} \times V_{u_{0}} \subset X \times \mathbb{R}$.

By construction, the integer $r$ (the order) associated with this auxiliary system is the same as the one of the given system $\Sigma$.

Moreover, the following flags $D$ and $D^{A}$ of integrable distributions over $V_{x_{0}}$ :

$$
\begin{aligned}
D_{0}(x) & =T_{x} X, D_{1}(x)=\operatorname{Ker}\left(d_{x} h(x)\right), \ldots, D_{r}(x)=D_{r-1}(x) \cap \operatorname{Ker}\left(d_{x} L_{f}^{r-1} h(x)\right) \\
D & =\left\{D_{0} \supset D_{1} \supset \ldots \supset D_{r}\right\} ;
\end{aligned}
$$

and

$$
\begin{aligned}
D_{0}^{A}(x) & =T_{x} X, D_{1}^{A}(x)=\operatorname{Ker}\left(d_{x} H(x)\right), \ldots, D_{r}^{A}(x)=D_{r-1}^{A}(x) \cap \operatorname{Ker}\left(d_{x} L_{F}^{r-1} H(x)\right) \\
D^{A} & =\left\{D_{0}^{A} \supset D_{1}^{A} \supset \ldots \supset D_{r}^{A}\right\},
\end{aligned}
$$

are equal.

Let us "prolong" the auxiliary flag $D^{A}$, in the following way:

$$
\begin{aligned}
D_{r+1}^{A}(x, \tilde{\varphi}) & =D_{r}^{A}(x) \cap \operatorname{Ker}\left(d_{x} L_{F}^{r} H_{1}(x, \tilde{\varphi})\right), \\
D_{i+1}^{A}(x, \tilde{\varphi}) & =D_{i}^{A}(x) \cap \operatorname{Ker}\left(d_{x} L_{F}^{i} H_{1}(x, \tilde{\varphi})\right), \\
D^{A}(\tilde{\varphi}) & =\left\{D_{0}^{A} \supset D_{1}^{A} \supset \ldots \supset D_{r}^{A} \supset D_{r+1}^{A}(\tilde{\varphi}) \supset \ldots \supset D_{l}^{A}(\tilde{\varphi})=D_{l+1}^{A}(\tilde{\varphi})\right\},
\end{aligned}
$$

where $l$ is the first integer such that $D_{l}^{A}(x, \tilde{\varphi})=D_{l+1}^{A}(x, \tilde{\varphi})$ at generic points.

Definition 9. The auxiliary flag $D^{A}(\tilde{\varphi})$ is regular on an open subset $U \subset X \times I$, if $D_{l}^{A}(\tilde{\varphi})=\{0\}$, and all the other $D_{i}^{A}(\tilde{\varphi})$ have constant rank first $n-2 i(i \leq r)$, second $n-r-i(r<i<l)$, third, $0(i \geq l=n-r)$; on this open set. 
Definition 10. The auxiliary flag $D^{A}(\tilde{\varphi})$ is uniform on an open subset $U \subset X \times I$, if it is regular, and independent of $\tilde{\varphi}$.

\subsection{Normal form for a uniform auxiliary flag (systems of type 2)}

Here, we consider a regular system $\Sigma$ of type 2 , with a uniform auxiliary flag over $X \times I$. The flag being integrable (in the sense that it is a flag of integrable distributions of constant rank), around each point of $X \times I$, we can find coordinates $x$ such that:

$$
\begin{aligned}
D_{l-1}^{A}(\tilde{\varphi}) & =\operatorname{Span}\left\{\frac{\partial}{\partial x_{n}}\right\}, \ldots, D_{r+1}^{A}(\tilde{\varphi})=\operatorname{Span}\left\{\frac{\partial}{\partial x_{n}}, \ldots, \frac{\partial}{\partial x_{2 r+2}}\right\}, \\
D_{r}^{A}(\tilde{\varphi}) & =\operatorname{Span}\left\{\frac{\partial}{\partial x_{n}}, \ldots, \frac{\partial}{\partial x_{2 r+1}}\right\}, D_{1}^{A}(\tilde{\varphi})=\operatorname{Span}\left\{\frac{\partial}{\partial x_{n}}, \ldots, \frac{\partial}{\partial x_{3}}\right\} .
\end{aligned}
$$

Moreover, we can take as $x$ coordinates:

$$
x_{1}=h_{1}(x), x_{2}=h_{2}(x), \ldots, x_{2 r-1}=L_{F}^{r-1} h_{1}(x), x_{2 r}=L_{F}^{r-1} h_{2}(x) .
$$

Then, the auxiliary system $\Sigma_{A}$ can be written:

$$
\begin{aligned}
\dot{x}_{1} & =x_{3}, \dot{x}_{2}=x_{4}, \\
& . . \\
\dot{x}_{2 r-3} & =x_{2 r-1}, \dot{x}_{2 r-2}=x_{2 r}, \\
\dot{x}_{2 r-1} & =F_{2 r-1}(x, \tilde{\varphi}), \dot{x}_{2 r}=F_{2 r}(x, \tilde{\varphi})=\tilde{\varphi}, \\
& . \\
\dot{x}_{n} & =F_{n}(x, \tilde{\varphi}), \\
y_{1} & =x_{1}, y_{2}=x_{2} .
\end{aligned}
$$

Since $D_{r+1}^{A}(\tilde{\varphi})=\operatorname{Span}\left\{\frac{\partial}{\partial x_{n}}, \ldots, \frac{\partial}{\partial x_{2 r+2}}\right\}$, we must have $\frac{\partial F_{2 r-1}}{\partial x_{2 r+2}}=\cdots=\frac{\partial F_{2 r-1}}{\partial x_{n}}=0$, and $\frac{\partial F_{2 r-1}}{\partial x_{2 r+1}} \neq 0$, or, locally:

$$
F_{2 r-1}=F_{2 r-1}\left(x_{1}, \ldots, x_{2 r+1}, \tilde{\varphi}\right), \frac{\partial F_{2 r-1}}{\partial x_{2 r+1}} \neq 0 .
$$

Repeating this reasoning, we get that:

$$
\begin{aligned}
F_{n-2} & =F_{n-2}\left(x_{1}, \ldots, x_{n-1}, \tilde{\varphi}\right), \frac{\partial F_{n-2}}{\partial x_{n-1}} \neq 0, \\
F_{n-1} & =F_{n-1}\left(x_{1}, \ldots, x_{n}, \tilde{\varphi}\right), \frac{\partial F_{n-1}}{\partial x_{n}} \neq 0 \\
F_{n} & =F_{n}(x, \tilde{\varphi})
\end{aligned}
$$

Conversely, if the system $\Sigma^{A}$ is like that, then it is just a trivial computation to check that the auxiliary flag is uniform.

Hence, replacing $\tilde{\varphi}$ by $\Phi(x, \varphi)=L_{f}^{r} h_{2}(x, \varphi)$, and reversing the role of $h_{1}, h_{2}$, we get the following result: 
Theorem 8 (Normal form for a uniform auxiliary flag). A system $\Sigma$ has a uniform auxiliary flag around $\left(x_{0}, \varphi_{0}\right)$, iff there is a neighborhood $V_{x_{0}} \times I_{\varphi_{0}}$ of $\left(x_{0}, \varphi_{0}\right)$, and coordinates on $V_{x_{0}}$ such that $\Sigma$ can be written:

$$
\begin{aligned}
y_{1} & =x_{1}, y_{2}=x_{2}, \\
\dot{x}_{1} & =x_{3}, \dot{x}_{2}=x_{4}, \\
& . \\
\dot{x}_{2 r-3} & =x_{2 r-1}, \dot{x}_{2 r-2}=x_{2 r}, \\
\dot{x}_{2 r-1} & =\Phi(x, \varphi), \dot{x}_{2 r}=F_{2 r}\left(x_{1}, \ldots, x_{2 r+1}, \Phi(x, \varphi)\right), \\
\dot{x}_{2 r+1} & =F_{2 r+1}\left(x_{1}, \ldots, x_{2 r+2}, \Phi(x, \varphi)\right), \\
. . & \\
\dot{x}_{n-1} & =F_{n-1}(x, \Phi(x, \varphi)), \\
\dot{x}_{n} & =F_{n}(x, \varphi),
\end{aligned}
$$

with $\frac{\partial \Phi}{\partial \varphi} \neq 0, \frac{\partial F_{2 r}}{\partial x_{2 r+1}} \neq 0, \ldots, \frac{\partial F_{n-1}}{\partial x_{n}} \neq 0$.

\subsection{Statement of the results for the two-output case}

Theorem 9 (main result in the 2-output case). If $\Sigma$ is uniformly infinitesimally identifiable (hence regular), then, there is an open-dense subanalytic subset $\tilde{U}$ of $X \times I$, such that at each point $\left(x_{0}, \varphi_{0}\right)$ of $\tilde{U}, \Sigma$ has the following properties, on a neighborhood of $\left(x_{0}, \varphi_{0}\right)$ :

- if $\Sigma$ has type 2, the auxiliary flag is uniform;

- if $\Sigma$ has type 1, then, $N(r)=n$.

Remark 8. a. In the case where $\Sigma$ has type 3, then, there is no other requirement.

b. In the case of type 1 assume $r=k$. Then, we have C., which implies $n>2 k$. But $N(r)=n=N(k)=2 k$. Then, for type 1 systems, $r=k$ cannot happen for a uniformly infinitesimally identifiable system.

This theorem is in fact equivalent to the theorem in the introduction, Theorem 4.

These two equivalent theorems (Ths. 4, 9) have a weak converse:

Theorem 10. Assume that $\Sigma$ satisfies the equivalent conditions of Theorems 4, 9, on some subset $V_{x_{0}} \times I_{\varphi_{0}}$ of $X \times I$ (so that, taking $V_{x_{0}}, I_{\varphi_{0}}$ small enough, the restriction $\Sigma_{\mid V_{x_{0}} \times I_{\varphi_{0}}}$ has one of the three normal forms above on $V_{x_{0}} \times I_{\varphi_{0}}$ ). Then, in case type 1, type 2 (normal forms 1.4, 1.5) $\Sigma_{\mid V_{x_{0}} \times I_{\varphi_{0}}}$ is uniformly infinitesimally identifiable and identifiable. In case type 3 (normal form 1.6), this is also true, eventually restricting the neighborhoods $V_{x_{0}}, I_{\varphi_{0}}$.

Also, in the special case of type 1 , there is a stronger result:

Theorem 11. Assume $\Sigma$ is uniformly infinitesimally identifiable (hence regular). Assume that $\Sigma$ has type 1. Then, there is an open-dense subanalytic subset $\tilde{X}$ of $X$, such that each point $x_{0}$ of $\tilde{X}$, has a neighborhood $V_{x_{0}}$, and coordinates $x$ on $V_{x_{0}}$ such that the system $\Sigma$ restricted to $V_{x_{0}} \times I$, denoted by $\Sigma_{\mid V_{x_{0}}}$, has the normal form 1.4 (globally over $V_{x_{0}} \times I$ ). Conversely, if it is the case, then, the restriction $\Sigma_{\mid V_{x_{0}}}$ is uniformly infinitesimally identifiable and identifiable.

Several points in this last theorem are not true in the case of types 2 and 3. 
Example 1. (Type 2) consider the type 2 system:

$$
\begin{aligned}
& y_{1}=x_{1}, y_{2}=x_{2}, \\
& \dot{x}_{1}=x_{3} \cos \varphi, \dot{x}_{2}=x_{3} \sin \varphi, \\
& \dot{x}_{3}=f(x) .
\end{aligned}
$$

where $x_{3}>0\left(x \in X=\mathbb{R}^{2} \times \mathbb{R}_{+}\right)$, and $I=[-A, A]$, for $A>0$, sufficiently large.

For this system, $k=1, r=1$, and $\mathbf{B}$. is always satisfied.

(a) The normal form 1.5 is met only locally with respect to $\varphi$ (changing the role of $h_{1}$ and $h_{2}$ depending on $\varphi$ ).

(b) For any open subset $\tilde{X}$ of $X, \Sigma_{\mid \tilde{X}}$ is never identifiable: for $\varphi(t)$ and $x(0)$ arbitrary, $(x(0), \varphi(t))$ and $(x(0), \varphi(t)+2 \pi)$ produce the same output.

Example 2. (Type 3) Consider the type 3 system on $\mathbb{R}^{2}$ :

$$
\begin{aligned}
& y_{1}=x_{1}, y_{2}=x_{2}, \\
& \dot{x}_{1}=\cos \varphi, \dot{x}_{2}=\sin \varphi,
\end{aligned}
$$

where, as above, $I=[-A, A]$, for $A>0$, sufficiently large. Again $(x(0), \varphi(t))$ and $(x(0), \varphi(t)+2 \pi)$ produce the same output.

Example 3. (Type 2) Consider the system on a subset $X \times I$ of $\mathbb{R}^{2} \times \mathbb{R}$, with $X$ open:

$$
\begin{aligned}
& y_{1}=\frac{1}{2}\left(\varphi-x_{2}\right)^{2}, y_{2}=x_{1} \\
& \dot{x}_{1}=x_{2}, \dot{x}_{2}=0 .
\end{aligned}
$$

For this system, $r=0, k=1$.

If we take for $X$ and $I$ neighborhoods of zero in $\mathbb{R}^{2}$ and $\mathbb{R}$, then, the auxiliary flag is not uniform (on any open subset of $X$ with zero in its closure), and the normal form 1.5 is not met. On the contrary, if $X$ is a neighborhood of $\left(x_{1}(0), x_{2}(0)\right)$, and $I$ is a neighborhood of $\varphi_{0}$, provided that these neighborhoods are small and $\varphi_{0} \neq x_{2}(0)$, the auxiliary flag is uniform, and the normal form is met.

\subsection{Proof of the results for the 2-output case}

First, let us show that Theorems 4,9 are equivalent.

It is just a matter of simple computations to see that normal form 1.5 (resp. 1.4, 1.6) in Theorem 4 imply the conditions "type 2" (resp. "type 1", "type 3" of Th. 9).

Conversely it is easy to check that, in Theorem 9, conditions "type 1", "type 3" imply the corresponding normal forms in Theorem 4. For type 3, see the (trivial) details in the proof of Theorems 4, 9, below. Condition "type 2 " is equivalent to the normal form 1.5 by Theorem 8 .

Proof of Theorem 10.

1. Normal form 1.4. From the normal form, computing the first variation, it follows immediately that $\hat{y}(t)=0$ on some time interval $[0, \varepsilon]$ ( $\hat{y}$ the output of the first variation), implies $\xi(0)=0$ on the same time interval, where $\xi(0) \in T_{x_{0}} X$ is the initial condition for the first variation. Deriving once more, we get that $\eta(\cdot)$ (the variation control) vanishes for almost all $t$. This shows that $\Sigma_{\mid V_{x_{0}} \times I_{\varphi_{0}}}$ is uniformly infinitesimally identifiable.

Now, from the normal form, differentiating the output $y(t)$ a certain number of times, one reconstructs the full state $x(t)=\left(x_{1}(t), \ldots, x_{n}(t)\right)$ of the system. 
Knowing $x(t)$, the equation $\dot{x}_{n}(t)=f_{n}(x(t), \varphi(t))$ can be solved with respect to $\varphi$, at almost all $t \in[0, \varepsilon]$ : if $\frac{\partial f_{n}}{\partial \varphi}$ never vanishes, then the function of $\varphi: \dot{x}_{n}(t)-f_{n}(x(t), \varphi)$ is a monotonous function of $\varphi$. Then, $\dot{x}_{n}(t)$ determines $\varphi$ uniquely, for almost all $t$. This shows that $\Sigma_{\mid V_{x_{0}} \times I_{\varphi_{0}}}$ is identifiable.

2. Normal form 1.6. Again, computing the first variation (with output $\hat{y}$ ), we see immediately that $\hat{y}(t)=0$ on some time interval $[0, \varepsilon]$ implies that $\xi(t)=0$ on this interval. The condition that $\left(\frac{\partial f_{n-1}}{\partial \varphi}, \frac{\partial f_{n}}{\partial \varphi}\right)$ does not vanish implies that $\eta(t)=0$ for almost all $t \in[0, \varepsilon]$ if $\hat{y}(\cdot)=0$. This shows that $\Sigma_{\mid V_{x_{0}} \times I_{\varphi_{0}}}$ is uniformly infinitesimally identifiable.

Now, from the normal form, any output trajectory $y(t)$ determines $x(t)$ by differentiation. The condition that $\left(\frac{\partial f_{n-1}}{\partial \varphi}, \frac{\partial f_{n}}{\partial \varphi}\right)$ does not vanish implies that (restricting the neighborhood $V_{x_{0}} \times I_{\varphi_{0}}$, and eventually exchanging the role of $\left.h_{1}, h_{2}\right)$ that $\frac{\partial f_{n-1}}{\partial \varphi}$ never vanishes. Then the same argument as for the normal form 1.4 shows that $\varphi(t)$ is determined almost everywhere by one more differentiation. $\Sigma_{\mid V_{x_{0}} \times I_{\varphi_{0}}}$ is identifiable.

3. Normal form 1.5. Computing with the first variation, assuming that the output $\hat{y}(t)$ is identically zero on some interval $[0, \varepsilon]$ we get that $\xi_{1}(t), \ldots, \xi_{2 r}(t)$ are identically zero on the same interval. The two equations:

$$
\dot{x}_{2 r-1}=\Phi(x, \varphi), \dot{x}_{2 r}=F_{2 r}\left(x_{1}, \ldots, x_{2 r+1}, \Phi(x, \varphi)\right),
$$

with $\frac{\partial \Phi}{\partial \varphi} \neq 0, \frac{\partial F_{2 r}}{\partial x_{2 r+1}} \neq 0$, show that $d_{x} \Phi . \xi(t)+d_{\varphi} \Phi . \eta(t)$ and $\xi_{2 r+1}(t)$ are identically zero for almost all $t$, and by continuity, $\xi_{2 r+1}(t)=0$ on $[0, \varepsilon]$. By induction, using the fact that $\frac{\partial F_{2 r+i}}{\partial x_{2 r+i+1}} \neq 0$, we get that $\xi_{2 r+i+1}(t)$ is identically zero, and at the end, $\xi(t)$ is identically zero.

The equation of $\xi_{2 r-1}$ again, shows that $\eta$ is zero almost everywhere: $0=d_{x} \Phi . \xi(t)+d_{\varphi} \Phi . \eta(t)$ a.e. Hence, $\Sigma$ is uniformly infinitesimally identifiable.

Now, the output $y(t)=\left(x_{1}, x_{2}\right)(t)$, by differentiation, determines $\left(x_{3}, x_{4}\right)(t)$ for all $t \in[0, \varepsilon]$. By differentiation of $\left(x_{3}, x_{4}\right)(t)$, we get $\left(x_{5}, x_{6}\right)(t)$, and so on. Once we know $\left(x_{2 r-1}, x_{2 r}\right)$, with the same reasoning, we determine $\Phi(x, \varphi)$ and $F_{2 r}\left(x_{1}, \ldots, x_{2 r+1}, \Phi(x, \varphi)\right)$ almost everywhere w.r.t. $t$. Now, $\frac{\partial F_{2 r}}{\partial x_{2 r+1}} \neq 0$ (never vanishes), shows that $F_{2 r}$ is monotonous w.r.t. $x_{2 r+1}$, the other variables being fixed. Hence, since we know $\Phi$ and $x_{1}, \ldots, x_{2 r}$, we can determine uniquely $x_{2 r+1}(t), t \in[0, \varepsilon]$ (by continuity) from the knowledge of the values of $F_{2 r}$ a.e. By induction, we determine $x(t)$ for all $t \in[0, \varepsilon]$.

Solving the equation $\dot{x}_{2 r-1}=\Phi\left(x(t), \varphi(t)\right.$ ) (using again the monotonicity of $\Phi$ w.r.t. $\varphi$, since $\frac{\partial \Phi}{\partial \varphi} \neq 0$ ), determines $\varphi(t)$ for almost all $t$, hence determines $\varphi$ as an $L_{\infty}\left([0, \varepsilon], I_{\varphi_{0}}\right)$ function. Hence, $\Sigma_{\mid V_{x_{0}} \times I_{\varphi_{0}}}$ is identifiable.

Proof of Theorem 11. Assume that $\Sigma$ is regular, type 1. Then, consider the subset $\tilde{X}$ of $X$ where

$$
d_{x} h_{1} \wedge d_{x} h_{2} \wedge d_{x} L_{f} h_{1} \wedge \ldots \wedge d_{x} L_{f}^{k-1} h_{1} \wedge d_{x} L_{f}^{k-1} h_{2} \neq 0
$$

$\tilde{X}$ is semi-analytic, open dense. On a neighborhood of each point of $\tilde{X}$, we can chose as coordinates $\left(x_{1}, x_{2}\right)=$ $h(x), \ldots,\left(x_{2 k-1}, x_{2 k}\right)=L_{f}^{k-1} h(x)$. Let us assume that $r>k$ or $\mathbf{C . 2}$ is satisfied (the case $\mathbf{C . 1}$ is obtained by 
exchanging the role of $h_{1}$ and $h_{2}$ ). Then, $\Sigma$ can be written locally in $x$ :

$$
\begin{aligned}
y_{1} & =x_{1}, y_{2}=x_{2}, \\
\dot{x}_{1} & =x_{3}, \dot{x}_{2}=x_{4}, \\
. . & \\
\dot{x}_{2 k-3} & =x_{2 k-1}, \dot{x}_{2 k-2}=x_{2 k}, \\
\dot{x}_{2 k-1} & =f_{2 k-1}\left(x_{1}, \ldots, x_{2 k+1}\right), \\
\dot{x}_{2 k} & =x_{2 k+1}, \\
. . & \\
\dot{x}_{N(r)-1} & =x_{N(r)}, \\
\dot{x}_{N(r)} & =f_{N(r)}(x, \varphi), \\
. . & \\
\dot{x}_{n} & =f_{n}(x, \varphi)
\end{aligned}
$$

with $\frac{\partial f_{N(r)}}{\partial \varphi}$ nonidentically zero.

Moreover, if $r=k$, then $f_{2 k-1}=f_{2 k-1}\left(x_{1}, \ldots, x_{2 k}\right)$, and $\dot{x}_{2 k}=f_{2 k}(x, \varphi)=f_{N(r)}(x, \varphi)$.

Assume that $N(r)<n$. Then, let us consider the initial condition $\xi(0)$ for the first variation: $\xi_{1}(0)=\cdots=$ $\xi_{N(r)}(0)=0, \xi_{N(r)+1}(0) \neq 0$. Chose the feedback function $\eta(x, \varphi, \xi)=-\frac{\mathrm{d}_{x} f_{N(r)}(x, \varphi) \xi}{\mathrm{d}_{\varphi} f_{N(r)}(x, \varphi)}$. For this, chose any function $\varphi$ ( $\varphi$ constant for instance). We have, for the first variation:

$$
\begin{aligned}
\dot{\xi}_{1} & =\xi_{3}, \dot{\xi}_{2}=\xi_{4} \\
& . \\
\dot{\xi}_{2 k-1} & =\frac{\partial f_{2 k-1}}{\partial x_{1}} \xi_{1}+\cdots+\frac{\partial f_{2 k-1}}{\partial x_{2 k+1}} \xi_{2 k+1}, \\
\dot{\xi}_{2 k} & =\xi_{2 k+1}, \\
. & \\
\dot{\xi}_{N(r)-1} & =\xi_{N(r)}, \\
\dot{\xi}_{N(r)} & =0 \text { by construction. }
\end{aligned}
$$

Moreover, if $r=k, \frac{\partial f_{2 k-1}}{\partial x_{2 k+1}} \equiv 0$, and $\dot{\xi}_{N(r)}=\dot{\xi}_{2 k}=0$.

Hence, for $t>0$ small, $\left(\xi_{1}(t), \xi_{2}(t)\right)=\hat{y}(t)=0$ (remind that $\hat{y}$ is the output of the first variation). This contradicts the uniform infinitesimal identifiability.

Hence, $N(r)=n$. Let $E=\left\{(x, \varphi) \mid d_{\varphi} L_{f}^{r} h(x, \varphi)=0\right\}$. Let $\pi E$ be the projection of $E$ on $X . X \backslash \pi E$ is subanalytic. Assume that $\pi E$ contains an open set $\tilde{X}$. By Hardt's theorem on the stratification of mappings, there is a smooth (analytic) mapping $\hat{\varphi}: \tilde{X} \rightarrow E$ (restricting $\tilde{X}$ eventually). Then, choosing for the first variation the initial condition $\xi(0)=0$, and any nonzero variation $\eta(t)$, but the feedback "control" $\varphi(t)=\hat{\varphi}(x(t)$ ), (for small times), we get by construction that the trajectory $\xi(t)$ of the first variation is in the zero section of $T X$. A contradiction with the uniform infinitesimal identifiability.

We conclude that $\pi E$ has codimension 1 , and $\tilde{X}=X \backslash \pi E$ contains an open dense set. This shows the first part of the theorem.

Since on $\tilde{X} \times I, d_{\varphi} L_{f}^{r} h(x, \varphi)$ never vanishes by construction, the proof of the last part of the theorem is exactly the same as the proof of Theorem 10, part "type 1".

Proof of Theorems 4, 9. We already know, by the beginning of this section, that these theorems are equivalent. 
The proof of Theorem 4, "type 1", is already contained in the proof of Theorem 11 (which is stronger, for type 1 systems).

Type 3 (the most simple case). It is clear that if $r=k, n=2 k$, the system can be locally written under normal form 1.6 around any point of an open dense semi-analytic subset of $X \times I: x_{1}=h_{1}(x), x_{2}=h_{2}(x), \ldots, x_{2 k-1}=$ $L_{f}^{k-1} h_{1}(x), x_{2 k}=L_{f}^{k-1} h_{2}(x)$ are adequate coordinates around any point where they are independent. The set of these points is semianalytic open, dense in $X$. The fact that $r=k$ implies that $d_{\varphi} L_{f}^{k} h(x, \varphi)$ is not identically zero. Hence, it is never zero on a semi-analytic open dense subset of $X \times I$. On the intersection of these two semianalytic subsets of $X \times I$ (the first one can be considered as such), in the coordinates just defined, the system is under the normal form 1.6.

Type 2. This is the most difficult case. In that case, by the definition of type 2, eventually interchanging $h_{1}, h_{2}$, we may assume that, around any point $\left(x_{0}, \varphi_{0}\right)$ of the complement $(X \times I) \backslash \tilde{U}$ of the codimension 1 analytic set $\tilde{U} \subset X \times I$ :

$$
\tilde{U}=\left\{(x, \varphi) \mid d_{x} h_{1} \wedge d_{x} h_{2} \wedge d_{x} L_{f} h_{1} \wedge \ldots \wedge d_{x} L_{f}^{r-1} h_{2} \wedge d_{x} L_{f}^{r} h_{1}=0\right\} \cup\left\{(x, \varphi) \mid d_{\varphi} L_{f}^{r} h_{2}=0\right\},
$$

we can find coordinates $x$ such that the system can be written:

$$
\begin{aligned}
y_{1} & =x_{1}, y_{2}=x_{2}, \\
\dot{x}_{1} & =x_{3}, \dot{x}_{2}=x_{4}, \\
. . & \\
\dot{x}_{2 r-3} & =x_{2 r-1}, \dot{x}_{2 r-2}=x_{2 r}, \\
\dot{x}_{2 r-1} & =f_{2 r-1}(x, \varphi), \dot{x}_{2 r}=f_{2 r}(x, \varphi), \\
. . & \\
\dot{x}_{n} & =f_{n}(x, \varphi),
\end{aligned}
$$

with $\frac{\partial f_{2 r}}{\partial \varphi} \neq 0$, and $d x_{1} \wedge \ldots \wedge d x_{2 r} \wedge d_{x} f_{2 r-1} \neq 0$.

Here, in case $r=k$, we treat B.1. only. In case $r<k$, we chose $h_{2}$ for $d_{\varphi} L_{f}^{r} h_{2} \neq 0$, and $h_{1}$ is the other. This ensures that $\tilde{U}$ has codimension 1 .

Let $\hat{y}(t)$ be an output function of the first variation of the system, which is identically zero on some time interval $[0, \varepsilon]$. Let $x(t), \xi(t), \varphi(t), \eta(t)$ be the corresponding trajectories. We know that the couple $(\xi(t), \eta(t))$ has to be identically zero, by the uniform infinitesimal identifiability. Differentiating $\hat{y}(t)=0 r$ times, we get:

$\xi_{1}(t)=\cdots=\xi_{2 r}(t)=0$ for all $t \in[0, \varepsilon]$, and:

$$
\begin{aligned}
d_{x} f_{2 r-1}(x(t), \varphi(t)) \xi(t)+d_{\varphi} f_{2 r-1}(x(t), \varphi(t)) \eta(t) & =0 \\
d_{x} f_{2 r}(x(t), \varphi(t)) \xi(t)+d_{\varphi} f_{2 r}(x(t), \varphi(t)) \eta(t) & =0
\end{aligned}
$$

for almost all $t \in[0, \varepsilon]$.

Hence, the system of equations (5.3), must have no smooth solution $(\eta, \varphi)(x, \xi)$, in a neighborhood of $\left(x_{0}, \xi(0)\right)$, $\xi(0) \neq 0$, in $X \times \mathbb{R}^{n-2 r}=\left\{(x, \xi(0)) \mid \xi_{1}(0)=\cdots=\xi_{2 r}(0)=0\right\}$, with $\varphi$ close to $\varphi_{0}$ and $\eta$ arbitrary:

Indeed, assume that there is a solution $(\eta, \varphi)(x, \xi)$. Then, consider the feedback system:

$$
\begin{aligned}
\dot{x} & =f(x, \varphi(x, \xi)) \\
\dot{\xi}_{2 r+1} & =d_{x} f_{2 r+1}(x, \varphi(x, \xi)) \xi+d_{\varphi} f_{2 r+1}(x, \varphi(x, \xi)) \eta(x, \xi), \\
& \ldots \\
\dot{\xi}_{n} & =d_{x} f_{n}(x, \varphi(x, \xi)) \xi+d_{\varphi} f_{n}(x, \varphi(x, \xi)) \eta(x, \xi),
\end{aligned}
$$

in which $\xi_{1}=\cdots=\xi_{2 r}=0$. 
This is a smooth differential equation on an open subset of $X \times \mathbb{R}^{n-2 r}$. It has a smooth solution $x(t), \xi(t)$. We set $\hat{\varphi}(t)=\varphi(x(t), \xi(t)), \hat{\eta}(t)=\eta(x(t), \xi(t))$, with $\xi(t) \neq 0$.

By construction, we have:

$$
\begin{aligned}
\dot{x} & =f(x, \hat{\varphi}(t)), \\
\dot{\xi} & =T_{x} f(x, \hat{\varphi}(t)) \xi+d_{\varphi} f(x, \hat{\varphi}(t)) \hat{\eta}(t),
\end{aligned}
$$

because, for $\xi_{1}, \ldots, \xi_{2 r}$, these equations read $\xi_{1}(t)=\cdots=\xi_{2 r}(t)=0$ for all $t$ (small).

Hence, in particular $\hat{y}_{1}(t)=0, \hat{y}_{2}(t)=0$. This is impossible, by uniform infinitesimal identifiability.

Therefore, by the crucial Lemma 7, Section A, we get that:

there are neighborhoods $V_{x_{0}}, V_{\varphi_{0}}$, and coordinates $\tilde{x}$ on $V_{x_{0}}$, with $\tilde{x}_{1}=x_{1}, \ldots, \tilde{x}_{2 r}=x_{2 r}$, such that:

$$
\tilde{x}_{2 r+1}=\Phi_{\varphi_{0}}\left(\tilde{x}_{1}, \ldots, \tilde{x}_{2 r}, f_{2 r-1}(\tilde{x}, \varphi), f_{2 r}(\tilde{x}, \varphi)\right)
$$

for all $(\tilde{x}, \varphi) \in V_{x_{0}} \times V_{\varphi_{0}}$.

Moreover,

$$
\begin{gathered}
\left(\frac{\partial \Phi_{\varphi_{0}}}{\partial f_{2 r-1}}, \frac{\partial \Phi_{\varphi_{0}}}{\partial f_{2 r}}\right) \text { never vanishes, } \\
\frac{\partial f}{\partial \varphi} \bar{\wedge} \frac{\partial f}{\partial \tilde{x}_{2 r+1}} \text { never vanishes, }
\end{gathered}
$$

on $V_{x_{0}} \times V_{\varphi_{0}}$, where $\frac{\partial f}{\partial \varphi} \bar{\wedge} \frac{\partial f}{\partial \tilde{x}_{p+1}}$ denotes the determinant of the $2 \times 2$ matrix formed by $\frac{\partial\left(f_{2 r-1}, f_{2 r}\right)}{\partial \varphi}$ and $\frac{\partial\left(f_{2 r-1}, f_{2 r}\right)}{\partial \tilde{x}_{2 r+1}}$.

In fact, $\frac{\partial \Phi_{\varphi_{0}}}{\partial f_{2 r-1}} \neq 0$, because, if it is zero, then $\frac{\partial \Phi_{\varphi_{0}}}{\partial f_{2 r}} \neq 0$ by (5.5), and, differentiating (5.4) with respect to $\varphi$, we get a contradiction (since $\frac{\partial f_{2 r}}{\partial \varphi} \neq 0$ ).

Therefore, we can apply the implicit function theorem to (5.4): restricting may be our neighborhood $V_{x_{0}} \times V_{\varphi_{0}}$, we find a smooth function $\bar{\Phi}$ such that

$$
f_{2 r-1}(\tilde{x}, \varphi)=\bar{\Phi}\left(\tilde{x}_{1}, \ldots, \tilde{x}_{2 r}, \tilde{x}_{2 r+1}, f_{2 r}(\tilde{x}, \varphi)\right)
$$

with $\frac{\partial \bar{\Phi}}{\partial \tilde{x}_{2 r+1}} \neq 0$.

Now, set $\Delta=\frac{\partial\left(f_{2 r-1}, f_{2 r}\right)}{\partial \varphi} \pi \frac{\partial\left(f_{2 r-1}, f_{2 r}\right)}{\partial \tilde{x}_{2 r+1}}$. An easy computation shows that $\Delta=-\frac{\partial f_{2 r}}{\partial \varphi} \frac{\partial \bar{\Phi}}{\partial \tilde{x}_{2 r+1}}$. This says no more than $\frac{\partial f_{2 r}}{\partial \varphi} \neq 0$, which we already know. Equation (5.3) becomes:

$$
\begin{aligned}
\text { 1. } \frac{\partial \bar{\Phi}}{\partial \tilde{x}_{2 r+1}} \xi_{2 r+1}+\frac{\partial \bar{\Phi}}{\partial f_{2 r}} d_{x} f_{2 r}(\tilde{x}, \varphi) \xi+\frac{\partial \bar{\Phi}}{\partial f_{2 r}} d_{\varphi} f_{2 r} \eta & =0, \\
d_{x} f_{2 r}(\tilde{x}, \varphi) \xi+d_{\varphi} f_{2 r} \eta & =0 .
\end{aligned}
$$


Equation $(5.8,2)$ implies $\eta=-\frac{d_{x} f_{2 r}(\tilde{x}, \varphi) \xi}{d_{\varphi} f_{2 r}}$, which replaced in $(5.8,1)$ gives $\frac{\partial \bar{\Phi}}{\partial \tilde{x}_{2 r+1}} \xi_{2 r+1}=0, \xi_{2 r+1} \equiv 0$. We have shown that $\hat{y} \equiv 0$ implies $\left(\xi_{1}, \ldots, \xi_{2 r+1}\right) \equiv 0$, and, making the change of notations $x:=\tilde{x},(5.2)$ rewrites:

$$
\begin{aligned}
y_{1} & =x_{1}, y_{2}=x_{2}, \\
\dot{x}_{1} & =x_{3}, \dot{x}_{2}=x_{4}, \\
& \ldots \\
\dot{x}_{2 r-3} & =x_{2 r-1}, \dot{x}_{2 r-2}=x_{2 r}, \\
\dot{x}_{2 r-1} & =f_{2 r-1}\left(x_{1}, \ldots, x_{2 r+1}, f_{2 r}(x, \varphi)\right), \dot{x}_{2 r}=f_{2 r}(x, \varphi), \\
\dot{x}_{2 r+1} & =f_{2 r+1}(x, \varphi), \\
& \ldots \\
\dot{x}_{n} & =f_{n}(x, \varphi),
\end{aligned}
$$

$\frac{\partial f_{2 r}}{\partial \varphi} \neq 0$, and $\frac{\partial f_{2 r-1}}{\partial x_{2 r+1}} \neq 0$. With $\xi_{1}(0)=\cdots=\xi_{2 r+1}(0)=0$, we obtain, for the first variation:

$$
\begin{aligned}
\hat{y}_{1} & =\xi_{1}, \hat{y}_{2}=\xi_{2}, \\
\dot{\xi}_{1} & =\xi_{3}, \dot{\xi}_{2}=\xi_{4} \\
& \ldots \\
\dot{\xi}_{2 r-3} & =\xi_{2 r-1}, \dot{\xi}_{2 r-2}=\xi_{2 r}, \\
\dot{\xi}_{2 r-1} & =d_{x} f_{2 r-1}\left(\xi_{1}, . ., \xi_{2 r+1}\right)+\frac{\partial f_{2 r-1}}{\partial f_{2 r}}\left(d_{x} f_{2 r} \xi+d_{\varphi} f_{2 r} \eta\right), \\
\dot{\xi}_{2 r} & =d_{x} f_{2 r} \xi+d_{\varphi} f_{2 r} \eta, \\
\dot{\xi}_{2 r+1} & =d_{x} f_{2 r+1} \xi+d_{\varphi} f_{2 r+1} \eta, \\
& \ldots \\
\dot{\xi}_{n} & =d_{x} f_{n} \xi+d_{\varphi} f_{n} \eta .
\end{aligned}
$$

If $n=2 r+1$, our theorem is already proved (exchanging $h_{1}, h_{2}$ ). Assume $n>2 r+1$.

If we can find a feedback solution $(\hat{\eta}, \hat{\varphi})(x, \xi)$ of :

$$
\begin{aligned}
d_{x} f_{2 r}(x, \varphi) \xi+d_{\varphi} f_{2 r}(x, \varphi) \eta & =0, \\
d_{x} f_{2 r+1}(x, \varphi) \xi+d_{\varphi} f_{2 r+1}(x, \varphi) \eta & =0
\end{aligned}
$$

for $\left(\xi_{1}, \ldots, \xi_{2 r+1}\right)=0,\left(\xi_{2 r+2}, \ldots, \xi_{n}\right) \neq 0$, on some open set in the space of the other variables $\xi_{2 r+2}, \ldots, \xi_{n}, x$, then, this will contradict the uniform infinitesimal identifiability, with the same reasoning as above. Therefore, another application of Lemma 7 shows that, we can change the coordinates $x$, keeping $x_{1}, \ldots, x_{2 r+1}$ unchanged, for:

$$
f_{2 r+1}=f_{2 r+1}\left(x_{1}, \ldots, x_{2 r+2}, f_{2 r}(x, \varphi)\right),
$$

where $\frac{\partial f_{2 r+1}}{\partial x_{2 r+2}} \neq 0$ (never vanishes on some neighborhood $V_{x_{0}} \times V_{\varphi_{0}}$ ). Iterating the process, and at the end, exchanging the roles of $y_{1}=x_{1}$ and $y_{2}=x_{2}$, ends the proof of the theorem.

\section{IDENTIFICATION}

We will not consider the case where identifiability is a generic property $\left(d_{y} \geq 3\right)$. In this case, there is a new difficulty, and it will be treated in another paper. For the cases $d_{y}=1, d_{y}=2$, we will be very short, and give only the ideas, leaving all details to the reader. Also, we will focus on the problem of "on line" identification 
(that is, the process of learning about the unknown function runs simultaneously to the process of observing the data $y(t))$.

In the case $d_{y}=1$, (normal form 1.3 ), the most simple example is:

$$
y^{(n)}=\varphi
$$

This trivial example shows that there is in general no hope to do something better than approximate differentiation: the problem of learning about the graph of $\varphi$ is just the problem of estimating the $n$ first derivatives of the output.

\subsection{Identification using approximate derivators}

Theorem 12. We consider, on $X \times I$, ( $X$ an open subset of $\left.\mathbb{R}^{n}\right)$, a system which is globally in normal form 1.3, $\left(d_{y}=1\right)$ or in normal forms 1.4, 1.5, $\left(d_{y}=2\right)$, or 1.6 with the additional requirement that $\frac{\partial f_{n}}{\partial \varphi}$ never vanishes $\left(d_{y}=2\right)$. The points of the graph of the functions $\varphi(x)$, or $\varphi \circ \pi(x)$ visited during some experiment can be reconstructed by (a finite number of) differentiations of the output(s).

Proof. Several facts in this theorem have been proved already. Nevertheless, we shortly prove everything.

\section{Normal forms $1.3,1.4,1.6$}

Differentiating the outputs, we can reconstruct the state $x(t)$ of the system. Differentiating once more, we reconstruct respectively $\psi(x, \varphi)(t)$ in $(1.3)$, and $f_{n}(x, \varphi)(t)$ in $(1.4,1.6)$. These functions, as functions of $\varphi$, are monotonous. Hence, $x(t)$ being known, we can reconstruct $\varphi(t)$ from the knowledge of their values.

\section{Normal form $\mathbf{1 . 5}$}

Differentiating a certain number of times, we reconstruct $x_{1}, \ldots, x_{2 r}$, and $\Phi(x, \varphi)$. Differentiating once more, we reconstruct $F_{2 r}\left(x_{1}, \ldots, x_{2 r+1}, \Phi\right)$. It is a monotonous function of $x_{2 r+1}$. Hence, $x_{2 r+1}$ can be obtained from these values. Iterating the result, we reconstruct $x(t)$. Once $x(t)$ is known, the function $\Phi$ being monotonous with respect to $\varphi$, we get $\varphi(t)$.

In fact, this procedure is not so far from what is done for the identification of linear systems.

\subsection{Identification using nonlinear observers}

We may assume, along the trajectories visited, a "local model" for $\varphi$ as a function of time. For instance, the most simple model is $\varphi^{(k)} \equiv 0$, i.e. $\varphi$ is a polynomial function of the time. Of course, the coefficients of this polynomial will be perpetually reestimated. And hence, the question is not that they model the function $\varphi$ globally as a function of time, but only locally, on reasonable time intervals (reasonable with respect to the performances of the observer).

Let us consider again the 4 normal forms 1.3-1.6 (with the same additional requirement, in case 1.6, that $\frac{\partial f_{n}}{\partial \varphi}$ never vanishes). Adding $\varphi$ and its $k$ first derivatives as extra state variables, the problem is now reduced to the problem of estimation of the state.

It turns out that, in all these cases, the extended systems we get have very strong observability properties, and that the "High Gain Construction", presented in the book [7], generalizes to these cases, allowing to reconstruct (approximately) the state of the extended system, and hence to estimate the corresponding points of the graph of $\varphi$.

\subsection{A more robust solution}

High gain observers may be rather sensitive to noise. A more robust solution is proposed in [3]. This construction also works for all the cases under consideration here in, if $d_{y}=1$ or 2 . 
As an example, let us consider just the case of the normal form (1.3), which gives, adding derivatives of $\varphi$ as state variables:

$$
\begin{aligned}
\frac{\mathrm{d}^{n} y}{\mathrm{~d} t^{n}} & =\psi\left(y, \ldots, y^{(n-1)}, \varphi\right), \\
\dot{\varphi} & =\varphi_{1}, \\
& . \\
\dot{\varphi}_{k-2} & =\varphi_{k-1}, \\
\dot{\varphi}_{k-1} & =0,
\end{aligned}
$$

where, as usual, $\frac{\partial \psi}{\partial \varphi}$ never vanishes.

The map $\Xi: \mathbb{R}^{n+k} \rightarrow \mathbb{R}^{n+k},\left(y, \dot{y}, \ldots, y^{n-1}, \varphi, \ldots, \varphi^{k-1}\right) \rightarrow\left(y, \dot{y}, \ldots, y^{(n+k-1)}\right)$, is a diffeomorphism, as it is easily checked. Hence (see again [7] for details), this system is diffeomorphic to a system on $\mathbb{R}^{n+k}$, of the form:

$$
\dot{\xi}_{1}=\xi_{2}, \ldots, \dot{\xi}_{n+k-1}=\xi_{n+k}, \dot{\xi}_{n+k}=\hat{\psi}(\xi), \quad y=\xi_{1},
$$

for some smooth function $\hat{\psi}$. This is exactly what is needed for applying the technique developed in [3].

We will exploit a variation of this idea for the biological reactor in the next section.

\section{The BIOLOGiCAL REACTOR}

\subsection{The model and its basic properties.}

Let us recall the equations of the model of bioreactor presented in the introduction:

$$
\begin{aligned}
& \frac{\mathrm{d} s}{\mathrm{~d} t}=-\mu \cdot x+D\left(S_{i n}-s\right) \\
& \frac{\mathrm{d} x}{\mathrm{~d} t}=(\mu-D) x .
\end{aligned}
$$

The growth function, $\mu(s, x)$, is smooth, positive or zero, and, for obvious physical reasons, $\mu(0, x)=0$ : if there is no substrate to eat, the population cannot grow. On the contrary, if there is something to eat, the population grows: $\mu(s, x)>0$ for $s>0$. The control function $D(t)$ verifies $D(t)>\varepsilon>0$ : this means that the reactor is always fed. The constant $S_{i n}$ is assumed to be strictly positive.

Now, the subset $X=\mathbb{R}^{+} \times \mathbb{R}^{+}$is invariant by the dynamics (7.1) of the bioreactor: if $x=0$, then $\dot{x}=0$, and if $s=0$, then $\dot{s}=D$. $S_{i n}>0$. Therefore, we may consider that $x(t), s(t)$ are always strictly positive.

The function $\mu(s, x)$ is often considered in the literature as a function of $s$ only, $\mu(0)=0, \mu \geq 0$. This means that the internal variable is $s \in \mathbb{R}^{+}$, and the internal mapping $\pi: X \rightarrow \mathbb{R}^{+}$is the mapping $(x, s) \rightarrow s$.

Typical expressions of the growth function in that case are the Monod model:

$$
\mu(s)=\frac{\mu_{0} s}{k_{m}+s},
$$

or the Haldane model:

$$
\mu(s)=\frac{\mu_{0} s}{k_{m}+s+\frac{s^{2}}{k_{i}}} .
$$




\subsection{Observation of $s$ only}

Setting $X=x+s$, we get:

$$
\begin{aligned}
\frac{\mathrm{d} s}{\mathrm{~d} t} & =-\mu \cdot x+D\left(S_{i n}-s\right), \\
\frac{\mathrm{d} X}{\mathrm{~d} t} & =D\left(S_{i n}-X\right),
\end{aligned}
$$

hence, setting $\tilde{D}(t)=\int_{0}^{t} D(\tau) \mathrm{d} \tau$, we get:

$$
X=\mathrm{e}^{-\tilde{D}(t)} X_{0}+\left(1-\mathrm{e}^{-\tilde{D}(t)}\right) S_{i n} .
$$

Let us set:

$$
\begin{aligned}
\text { (1) } \Lambda(t) & =\mathrm{e}^{\tilde{D}(t)}\left(s-S_{i n}\right)+S_{i n}, \quad \text { or: } \\
(2) \quad s & =\mathrm{e}^{-\tilde{D}(t)} \Lambda(t)+S_{i n}\left(1-\mathrm{e}^{-\tilde{D}(t)}\right), \quad s(0)=\Lambda(0)
\end{aligned}
$$

By (7.4), we get:

$$
\dot{\Lambda}=-\mathrm{e}^{\tilde{D}(t)}(X-s) \mu,
$$

and with (7.5):

$$
\dot{\Lambda}=\left(\Lambda-X_{0}\right) \mu .
$$

Let us assume, as we said in the previous section, that $\mu$ is a function of $s$ only. Let us also assume that $s(\cdot)$ visits twice the same value, i.e., $T_{0}<T_{1}, s\left(T_{0}\right)=s\left(T_{1}\right)$. Then, with $X_{0}=X(0)=x(0)+s(0)$ :

$$
\mu\left(s\left(T_{0}\right)\right)=\frac{\dot{\Lambda}\left(T_{0}\right)}{\Lambda\left(T_{0}\right)-X_{0}}=\frac{\dot{\Lambda}\left(T_{1}\right)}{\Lambda\left(T_{1}\right)-X_{0}}=\mu\left(s\left(T_{1}\right)\right) .
$$

Observe, with equation (7.8), that $\Lambda$ is everywhere continuously differentiable, even if $D(\cdot)$ is only measurable, bounded. From (7.7), and the fact that $\mu(s)>0$ for $s>0$, we get that $\Lambda(t)$ is a strictly decreasing function, and $\Lambda(0)=s(0), X_{0}=x(0)+s(0), x(0)>0$, implies that $\Lambda(t)-X_{0}$ is never zero for $t \geq 0$.

Also, $s(t)$ being observed, and $D(t)$, the control, being known, we may consider, by the definition $(7.6,1)$ of $\Lambda$, that $\Lambda$ is an observed function. Then, $X_{0}$ can be computed:

$$
X_{0}=\frac{\dot{\Lambda}\left(T_{0}\right) \Lambda\left(T_{1}\right)-\dot{\Lambda}\left(T_{1}\right) \Lambda\left(T_{0}\right)}{\dot{\Lambda}\left(T_{0}\right)-\dot{\Lambda}\left(T_{1}\right)},
$$

indeed, $\dot{\Lambda}\left(T_{0}\right)-\dot{\Lambda}\left(T_{1}\right)=\left(\Lambda\left(T_{0}\right)-\Lambda\left(T_{1}\right)\right) \mu\left(s\left(T_{0}\right)\right) \neq 0$.

Now, $X_{0}$ being known,

$$
\mu(s(t))=\frac{\dot{\Lambda}(t)}{\Lambda(t)-X_{0}}
$$

since $\Lambda(t)-X_{0}$ never vanishes.

Conversely, let us consider a trajectory, defined on $[0, T]$, such that:

(H.a.) $s$ never visits twice the same value on $[0, T]$ (i.e. $s$ is strictly monotonous),

or, stronger

(H.b.) $D(\cdot)$ is smooth, (from what it follows that $s(\cdot), x(\cdot), \Lambda(\cdot), X(\cdot)$, are all smooth functions), and $\frac{d s}{d t}(t) \neq$ 0 for all $t \in[0, T]$.

Initial conditions corresponding to this trajectory are $s_{0}, x_{0}, X_{0}$ (all strictly $\left.>0\right)$. 
Let us consider another arbitrary value, $\tilde{X}_{0}=\tilde{x}_{0}+s_{0}>0$, close to $X_{0}$. Then, since $\Lambda(t)=\mathrm{e}^{\tilde{D}(t)}\left(s-S_{i n}\right)+S_{\text {in }}$ is $C^{1}$ (even in case (H.a.)), as we know by (7.8), we may compute $\tilde{\mu}(t)=\frac{\dot{\Lambda}(t)}{\Lambda(t)-\tilde{X}_{0}}$, on the interval $[0, T]$ : indeed, since $\tilde{X}_{0}$ is close to $X_{0}$, then $\Lambda(t)-\tilde{X}_{0}$ is close to $\Lambda(t)-X_{0}$, and then never vanishes. Then, under assumption (H.a.), there is a continuous function $t(s)$, which is the inverse of $s(t)$, and which is smooth under assumption (H.b.). Set $\bar{\mu}(s)=\tilde{\mu}(t(s))$. In case (H.b.), $\bar{\mu}$ is smooth, in case (H.a.), it is continuous only. Set also $\tilde{x}(t)=\tilde{X}(t)-s(t)=\mathrm{e}^{-\tilde{D}(t)} \tilde{X}_{0}+\left(1-\mathrm{e}^{-\tilde{D}(t)}\right) S_{i n}-s(t)$. In these circumstances, we claim that:

Claim. (a) $s(t), \tilde{x}(t)$ are solutions of the system:

$$
\begin{aligned}
& \text { (1) } \frac{\mathrm{d} s}{\mathrm{~d} t}=-\bar{\mu}(s(t)) \tilde{x}+D(t)\left(S_{\text {in }}-s(t)\right) \\
& \text { (2) } \frac{\mathrm{d} \tilde{x}}{\mathrm{~d} t}=(\bar{\mu}(s(t))-D(t)) \tilde{x}(t)
\end{aligned}
$$

(b) if $\tilde{X}_{0}$ is sufficiently close to $X_{0}, \tilde{x}(t)>0$ for all $t \in[0, T]$.

Proof of the claim (a). Let us show first that $\mu(t) x(t)=\tilde{\mu}(t) \tilde{x}(t)$ for all $t \in[0, T]$. By construction:

$$
\tilde{\mu}(t)=\frac{\Lambda(t)-X_{0}}{\Lambda(t)-\tilde{X}_{0}} \mu(t)
$$

then it is sufficient to check that $\frac{\Lambda(t)-X_{0}}{\Lambda(t)-\tilde{X}_{0}} \mu(t) \tilde{x}(t)=\mu(t) x(t)$, or, since $\mu(t)>0$ :

$$
\left(\Lambda(t)-X_{0}\right) \tilde{x}(t)=\left(\Lambda(t)-\tilde{X}_{0}\right) x(t) .
$$

But $x(t)=X(t)-s(t)=\mathrm{e}^{-\tilde{D}(t)} X_{0}+\left(1-\mathrm{e}^{-\tilde{D}(t)}\right) S_{i n}-s(t)$, and $\tilde{x}(t)=\tilde{X}(t)-s(t)=\mathrm{e}^{-\tilde{D}(t)} \tilde{X}_{0}+(1-$ $\left.\mathrm{e}^{-\tilde{D}(t)}\right) S_{i n}-s(t)$. Replacing by these expressions and by the expression $(7.6,1)$ of $\Lambda(t)$, just shows that $(7.12)$ is true. Therefore, since $\frac{\mathrm{d} s}{\mathrm{~d} t}=-\mu \cdot x+D\left(S_{i n}-s\right)$, we get that also, $\frac{\mathrm{d} s}{\mathrm{~d} t}=-\tilde{\mu} . \tilde{x}+D\left(S_{i n}-s\right)$. This is (a, 1$)$.

Now,

$$
\begin{aligned}
\tilde{x}(t) & =\tilde{X}(t)-s(t)=\mathrm{e}^{-\tilde{D}(t)} \tilde{X}_{0}+\left(1-\mathrm{e}^{-\tilde{D}(t)}\right) S_{i n}-s(t), \\
\frac{\mathrm{d} \tilde{x}}{\mathrm{~d} t}(t) & =-D(t)\left(\tilde{x}(t)-S_{i n}+s(t)\right)+\mu(t) x(t)-D(t)\left(S_{i n}-s(t)\right), \\
& =-D(t) \tilde{x}(t)+\mu(t) x(t)=-D(t) \tilde{x}(t)+\tilde{\mu}(t) \tilde{x}(t),
\end{aligned}
$$

by the proof of $(\mathrm{a}, 1)$. Hence, $\frac{\mathrm{d} \tilde{x}}{\mathrm{~d} t}(t)=(\tilde{\mu}(t)-D(t)) \tilde{x}(t)$. This is $(\mathrm{a}, 2)$.

To prove (b), let us just observe that: $\tilde{x}(t)=\mathrm{e}^{-\tilde{D}(t)} \tilde{X}_{0}+\left(1-\mathrm{e}^{-\tilde{D}(t)}\right) S_{i n}-s(t)$, and $x(t)=\mathrm{e}^{-\tilde{D}(t)} X_{0}+(1-$ $\left.\mathrm{e}^{-\tilde{D}(t)}\right) S_{i n}-s(t)$, then, $\tilde{x}(t)-x(t)=\mathrm{e}^{-\tilde{D}(t)}\left(\tilde{X}_{0}-X_{0}\right)$. Hence, for $\tilde{X}_{0}$ sufficiently close to $X_{0}$, or equivalently $\tilde{x}_{0}$ sufficiently close to $x_{0}, \tilde{x}(t)$ is strictly positive.

We have shown the following theorem (more precise version of Th. 6 in Sect. 2.3):

Theorem 13. a. The bioreactor is identifiable at any admissible i-o trajectory $(s(t), D(t)), t \in[0, T]$ such that $s(t)$ visits twice the same value,

b. Conversely, if $(s(t), D(t)), t \in[0, T]$ is an admissible i-o trajectory along which $s(t)$ is strictly monotonous, then, there is an infinity of corresponding couples $(x(\cdot), \mu)$, with $\mu$ continuous. If moreover $D(\cdot)$ is smooth, and $\dot{s}(t) \neq 0$ for all $t \in[0, T]$, then, there is an infinity of corresponding couples $(x(\cdot), \mu)$, with $\mu$ smooth.

In Section 7.5, some numerical investigation will be made on the basis of this theorem. 


\subsection{Observation of both $s$ and $x$}

In that case, assuming $D(\cdot)$ constant, we are in the situation $d_{y}=2$, of Section 5 , and our system is regular, and type 3 (normal form 1.6), with the additional requirement of Theorem 12.

Therefore, we may use the ideas explained briefly in Section 6, Sections 6.2, 6.3. In fact, in that case, we can do better:

1. we can adapt these ideas to the case where $D(\cdot)$ is not constant;

2. making a small change of variables, we can use a standard linear Kalman observer, in place of a high-gain one.

Let us set $z(t)=\mu(s(t)) . x(t)$, and let us, (as explained in Sect. 6), assume a local model for $z(\cdot)$, of the form $\frac{\mathrm{d}^{k} z}{\mathrm{~d} t^{k}}=0$. Then, our system becomes:

$$
\begin{aligned}
\dot{s} & =-z+D(t)\left(S_{i n}-s\right) \\
\dot{x} & =z-D(t) \cdot x \\
\dot{z} & =z_{1}, \ldots, \dot{z}_{k-2}=z_{k-1}, \dot{z}_{k-1}=0 ; \\
y_{1} & =s, \quad y_{2}=x .
\end{aligned}
$$

Assuming only that $0 \leq D(t) \leq D_{\max }$, this is a linear time-dependant system, which is uniformly observable in the sense of the linear theory. Hence, classical versions of the (time dependant) Linear Kalman Filter work, even in a stochastic context.

This method will be also investigated numerically in Section 7.5.

\subsection{Observation of $x$ only}

This case is often considered in practice.

If $\mu$ would be a function $\mu(x)$ of $x$, then, we could consider the system:

$$
\begin{aligned}
& \dot{x}=(\mu(x)-D) x, \\
& y=x,
\end{aligned}
$$

for $x \in \mathbb{R}^{+}$. Then, assuming $D(\cdot)$ constant, we are exactly in the case of uniformly infinitesimally identifiable systems, in the case $d_{y}=1$, normal form 1.3. Our ideas of Section 6 can then be used, and work as perfectly as in the case of the previous Section 7.3 (even for $D(\cdot)$ non constant).

But, in the case where $\mu$ depends only on $s$, as we assume here (and as is often assumed in the literature), or if $\mu=\mu(s, x)$ depends on both $s$ and $x$, then, the system:

$$
\begin{aligned}
\dot{x} & =(\mu-D) x, \\
\dot{s} & =-\mu \cdot x+D\left(S_{i n}-s\right), \\
y & =x
\end{aligned}
$$

is not uniformly infinitesimally identifiable (it does not verify the necessary conditions of Th. 2).

Moreover, clearly, it is not identifiable: assume $D$ constant (for simplicity only), and $\mu(x, s)$, or $\mu(s)$ given, smooth. Given a smooth trajectory $(x(t), s(t)), t \in[0, T], x(0)>0, s(0)>0$, of this system. Then,

$$
s(t)=\mathrm{e}^{-D t} s(0)+\int_{0}^{t} \mathrm{e}^{-D(t-\tau)}\left(-(\mu x)(\tau)+D S_{i n}\right) \mathrm{d} \tau
$$


Let us chose another $\tilde{s}_{0} \neq s(0),(\tilde{s}(0)$ close to $s(0))$, and consider:

$$
\tilde{s}(t)=\mathrm{e}^{-D t} \tilde{s}_{0}+\int_{0}^{t} \mathrm{e}^{-D(t-\tau)}\left(-(\mu x)(\tau)+D S_{i n}\right) \mathrm{d} \tau .
$$

Consider now the trajectory $(x(t), \tilde{s}(t)), t \in[0, T]$, and a smooth function $\tilde{\mu}(x, s)$, or $\tilde{\mu}(s)$, such that $\tilde{\mu}(x(t), \tilde{s}(t))=$ $\mu(x(t), s(t))$ for $t \in[0, T]$. This is possible, eventually restricting the interval $[0, T]$ to some $\left[T_{0}, T_{1}\right] \subset[0, T]$. In fact, $(x(t), \tilde{s}(t))$ is a solution of:

$$
\begin{aligned}
& \frac{\mathrm{d} x}{\mathrm{~d} t}=(\tilde{\mu}-D) x, \\
& \frac{\mathrm{d} \tilde{s}}{\mathrm{~d} t}=-\tilde{\mu} \cdot x+D\left(S_{i n}-\tilde{s}\right),
\end{aligned}
$$

$x(0)=x_{0}, \tilde{s}(0)=\tilde{s}_{0}$.

Hence, some people identify systems that are not identifiable: it is possible, by differentiation of the output $x$, to obtain complete information on the function $\mu(x(t), s(t))$, as a function of time. But it is not possible to deduce from this, any information about the function $\mu(s, x)$, or $\mu(s)$.

In fact, the reason why they obtain "some results" in practice (even for $D(\cdot)$ nonconstant) is the following: because of equation (7.5), we see that, whatever the control $D(\cdot)>\varepsilon>0, X(t)$ tends to $S_{\text {in }}$ when $t \rightarrow$ $+\infty$. Hence, after some time, $X=x+s$ is close to $S_{i n}$, and $s$ is close to $S_{i n}-x$. Then, the estimate $\hat{\mu}(t)$, obtained after differentiation of the output $x(t)$, is such that $\left(x(t), S_{i n}-x(t), \hat{\mu}(t)\right)$, is close to a point of the graph of $\mu$, for $t$ large enough.

\subsection{Numerical simulations}

\subsubsection{Simulation with complete measurements}

In this section, we simulate the bioreactor model using the Monod growth function (7.2)

$$
\mu(s)=\frac{0.15 s}{2+s} .
$$

We assume $S_{\text {in }}$ constant, equal to $5 . D(t)$ is a periodic function with period shown in Figure 1. Initial conditions are $x(0)=3$ and $s(0)=2$. In this first case, we assume that both the substrate and the biomass concentration are measured. Hence the output is $y(t)=(s(t), x(t))$. A colored noise is added to both variables to simulate noisy measurement. More precisely, this colored noise has been simulated using

$$
\mathrm{d} U_{t}=-a U_{t} \mathrm{~d} t+\sigma \sqrt{2 a} \mathrm{~d} W_{t}
$$

where $W_{t}$ is a normalized Brownian motion. So $U_{t}$ is an Ornstein-Uhlenbeck process, that is, a stationary process with mean 0 and with covariance function

$$
\Gamma_{U}(t, s)=E\left[U_{t} U_{s}\right]=\sigma^{2} \mathrm{e}^{-a|t-s|}
$$

and hence $U_{t}$ is a reasonable approximation of a realistic noise.

As explained in Section 7.3, we set $z(t)=\mu(s(t)) x(t)$, and we assume a local model for $z(t)$ of the form

$$
\left\{\begin{array}{l}
\frac{\mathrm{d} z(t)}{\mathrm{d} t}=z_{1}(t) \\
\frac{\mathrm{d} z_{1}(t)}{\mathrm{d} t}=z_{2}(t) \\
\frac{\mathrm{d} z_{2}(t)}{\mathrm{d} t}=0 .
\end{array}\right.
$$




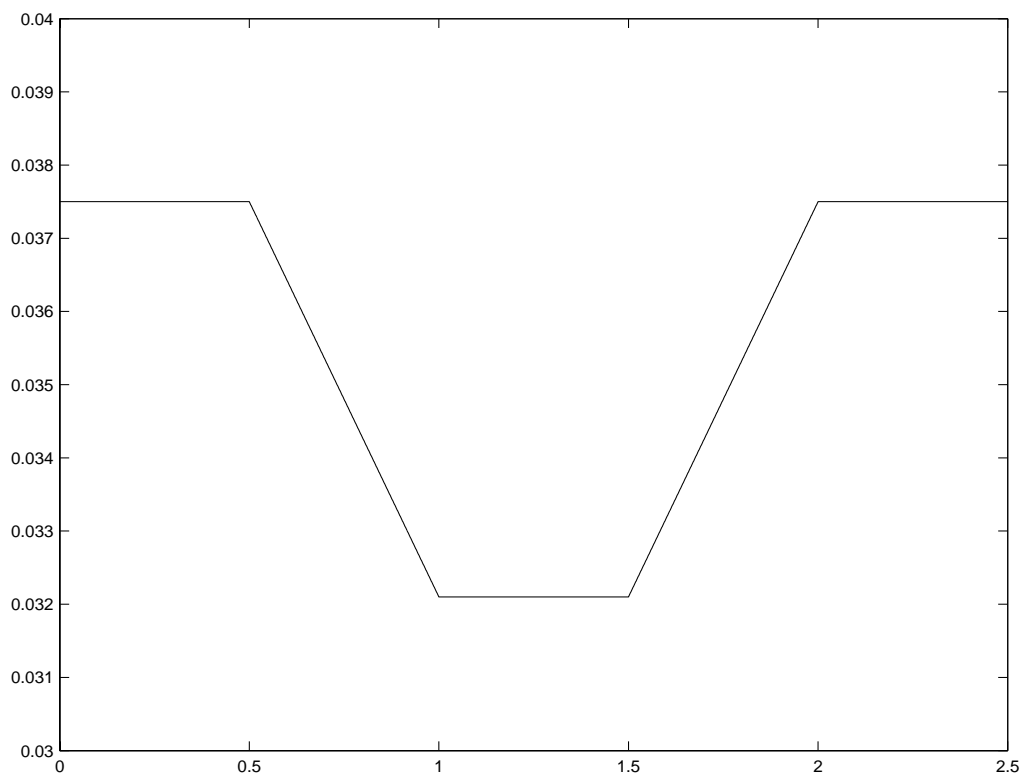

Figure 1. $D(t)$.

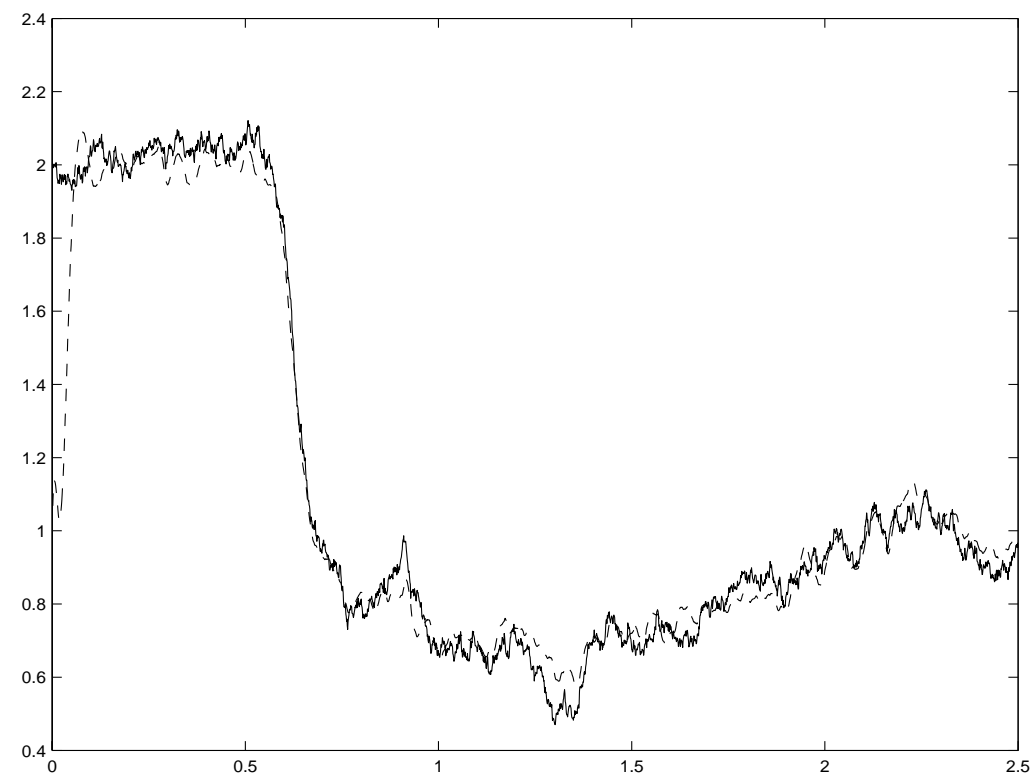

FIGURE 2. $s(t)$.

We add to these equations the two equations of the bioreactor

$$
\left\{\begin{array}{l}
\frac{\mathrm{d} s(t)}{\mathrm{d} t}=-z(t)+D(t)\left(S_{\mathrm{in}}-s(t)\right) \\
\frac{\mathrm{d} x(t)}{\mathrm{d} t}=z(t)-D(t) x(t)
\end{array}\right.
$$




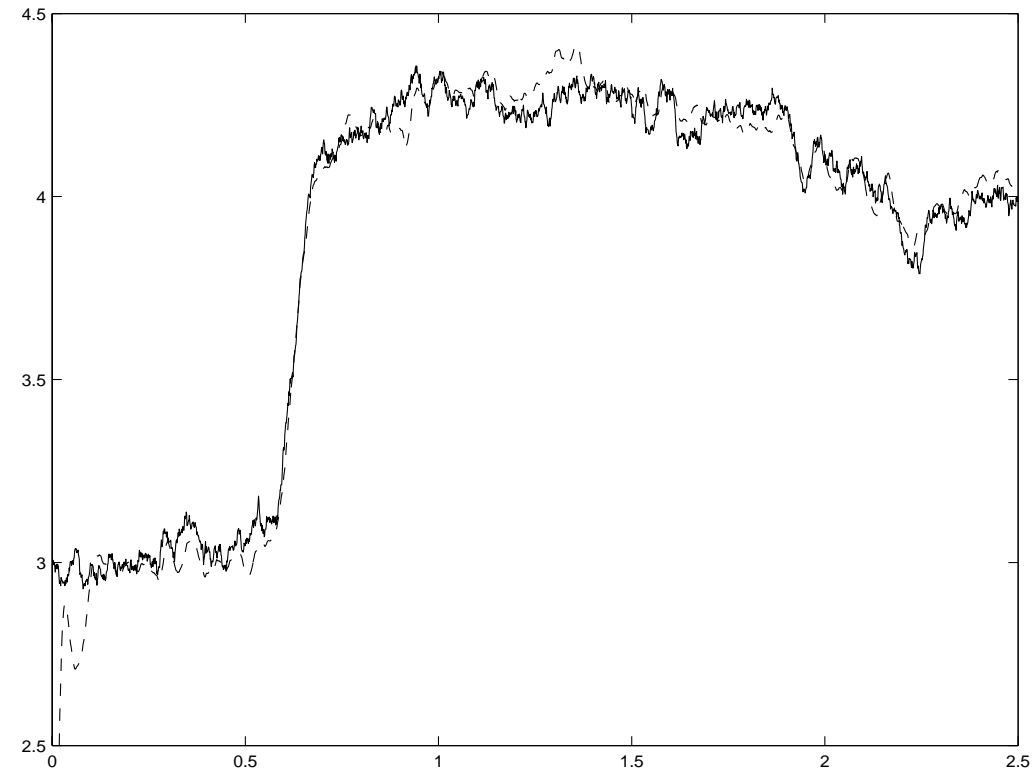

Figure 3. $x(t)$.

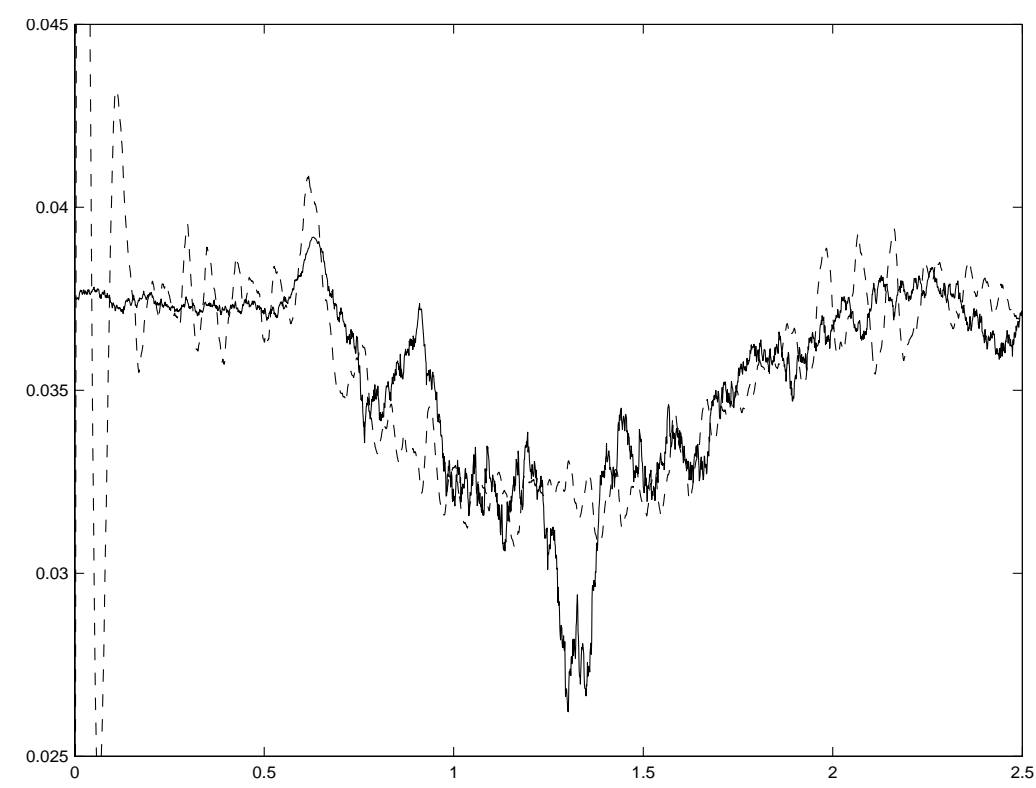

Figure 4. $\mu(t)$.

and we apply the classical Kalman filter to these five equations, i.e. setting $X(t)=\left(s(t), x(t), z(t), z_{1}(t), z_{2}(t)\right)$

$$
\begin{aligned}
& \frac{\mathrm{d} \widehat{X}(t)}{\mathrm{d} t}=A(t) \widehat{X}(t)+b(t)+P C^{T} R^{-1}(y(t)-C \widehat{X}(t)) \\
& \frac{\mathrm{d} P(t)}{\mathrm{d} t}=A(t) P(t)+P(t) A(t)^{T}+Q-P(t) C^{T} R^{-1} C P(t)
\end{aligned}
$$


with

$$
\begin{aligned}
& A(t)=\left(\begin{array}{ccccc}
-D(t) & 0 & -1 & 0 & 0 \\
0 & -D(t) & 1 & 0 & 0 \\
0 & 0 & 0 & 1 & 0 \\
0 & 0 & 0 & 0 & 1 \\
0 & 0 & 0 & 0 & 0
\end{array}\right), b(t)=\left(\begin{array}{c}
D(t) s_{\text {in }} \\
0 \\
0 \\
0 \\
0
\end{array}\right) \\
& \text { and } C=\left(\begin{array}{lllll}
1 & 0 & 0 & 0 & 0 \\
0 & 1 & 0 & 0 & 0
\end{array}\right) \text {. }
\end{aligned}
$$

Measured outputs are shown in Figures 2 and 3 where the continuous line represents noisy outputs and the dashed line represents observer estimation.

Figure 4 represents $\mu(t)$ and $\widehat{\mu}(t)=\frac{\widehat{z}(t)}{\widehat{x}(t)}$ with the same convention as in the previous figures. Clearly, after the initial transient, the behavior of the Kalman filter looks good.

7.5.2. Simulation with a single measurement $s(t)$

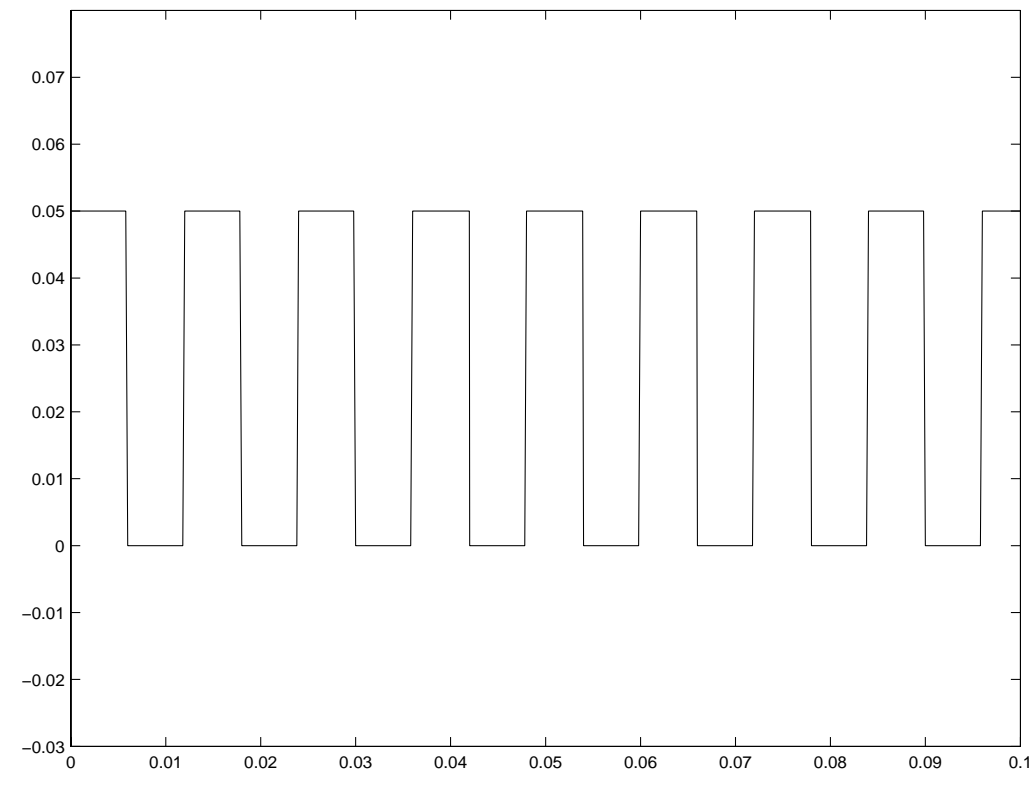

FiguRe 5. $D(t)$.

If $s$ is the only measurement, we can still expect to reconstruct $\mu(s)$ for all visited value of $s$ provided that at least one value of $s$ has been visited twice, as already explained in Section 7.2.

Since this identification process does not depend on the estimation of $x$, we use the estimation of $\mu$ to estimate $x$. Moreover, once $\mu$ is known, the system is a linear system with a matrix depending on the output $s$, therefore we can apply linear Kalman filter to estimate $x$.

Our algorithm is divided in two steps:

- estimation of $\mu$ using the redundant visited values of the measurement $s$. The estimation of the function $s \rightarrow \mu(s)$ at time $t$ is denoted by $s \rightarrow \widehat{\mu_{t}}(s)$;

- estimation of $x(t)$ on the basis of $s(t)$ and $\widehat{\mu_{t}}$.

The second part of our algorithm is simply standard Kalman filtering. Let us explain the first part. 


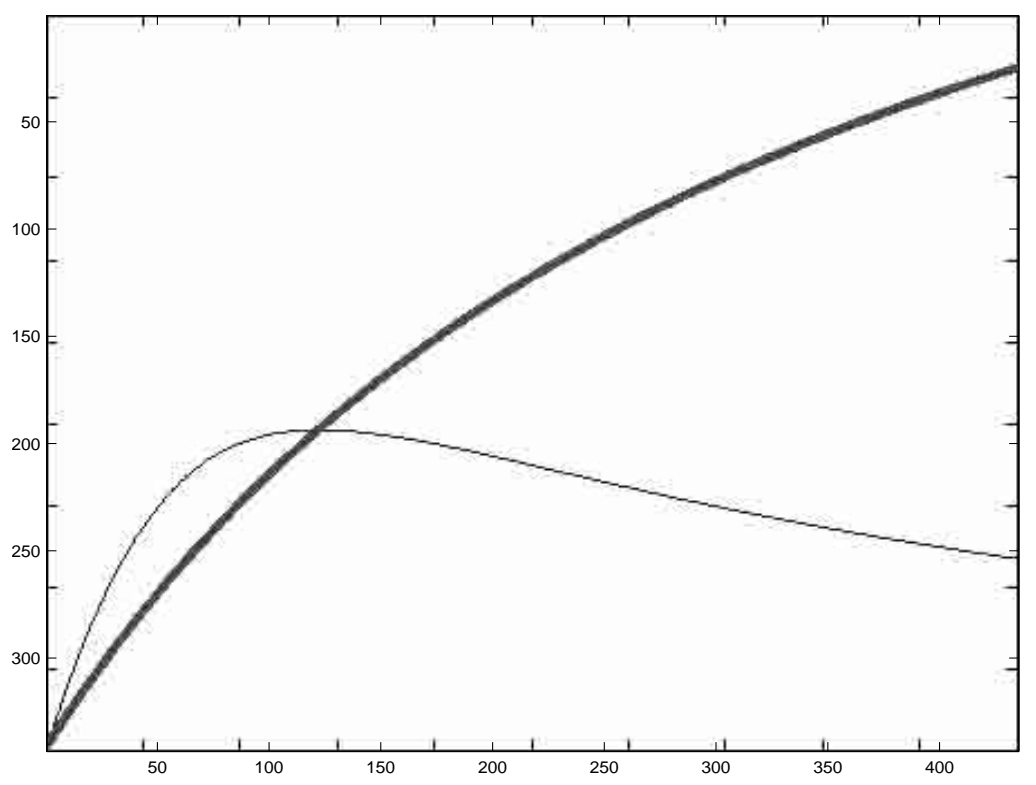

FigurE 6. $\mu(s)$ (thin line) and its initial guess $\widehat{\mu_{0}}(s)$ (thick line).

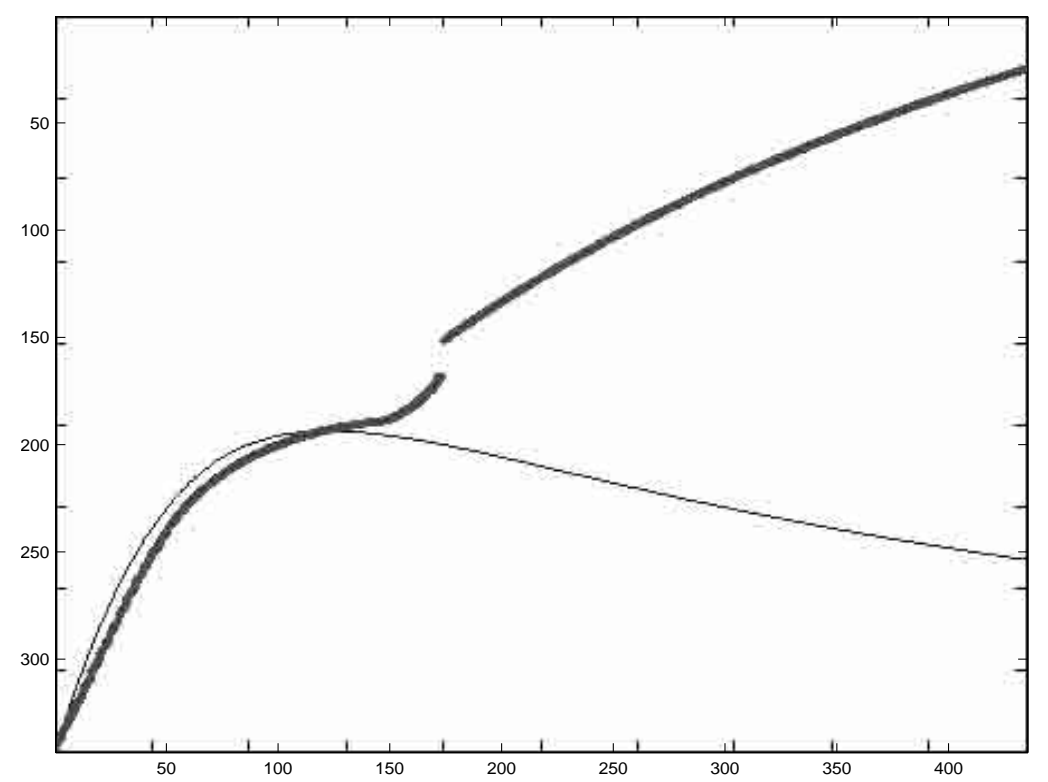

FiguRE 7. $\mu(s)$ and $\widehat{\mu_{t}}(s)$ at time $t=0.019$.

We first choose a sample time $\Delta t$, arbitrarily fixed to 0.2 . At each sample time $t=k \Delta t$, we consider the $N+1$ values of $s$ at time $k \Delta t,(k-1) \Delta t, \ldots,(k-N) \Delta t$, i.e. we consider only the history up to time $T=N \Delta t$ in the past.

Since we want to estimate a function, we have to discretize the coordinate space of $s$ in a range corresponding to physical values, with a small enough discretization step, to ensure good accuracy. In this way, we chose $s_{\text {min }}$ and $s_{\max }$ and a step size $\Delta s$. 


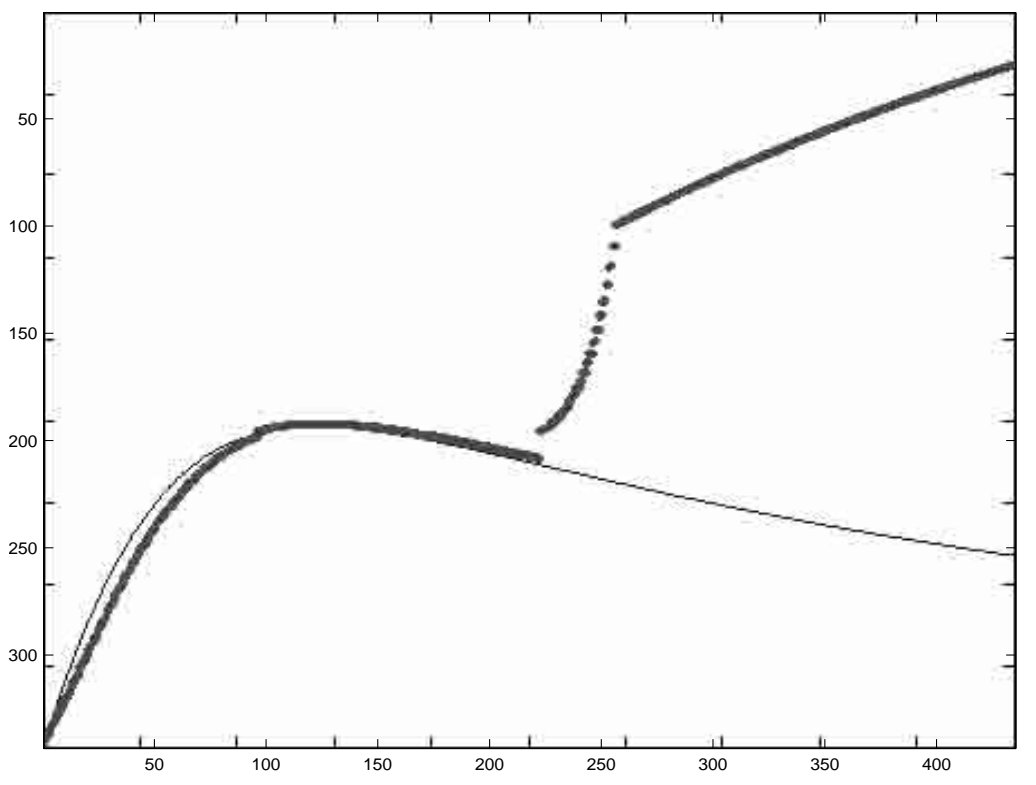

Figure 8. $\mu(s)$ and $\widehat{\mu_{t}}(s)$ at time $t=0.042$.

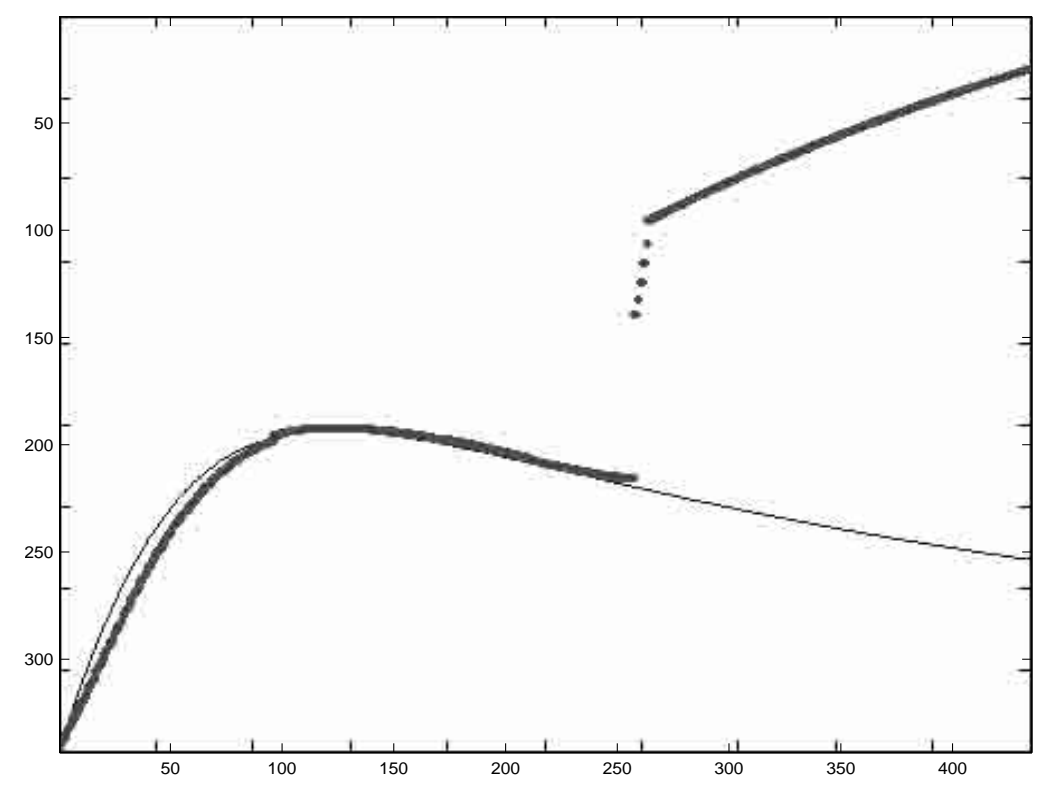

FiguRE 9. $\mu(s)$ and $\widehat{\mu_{t}}(s)$ at time $t=0.051$.

At each sample time $k \Delta t$, we replace the measured trajectory

$$
s((k-N) \Delta t), \ldots, s(k \Delta t)
$$

by a linearly interpolated trajectory

$$
s\left(t_{1}\right), s\left(t_{2}\right), \ldots, s\left(t_{n}\right)
$$




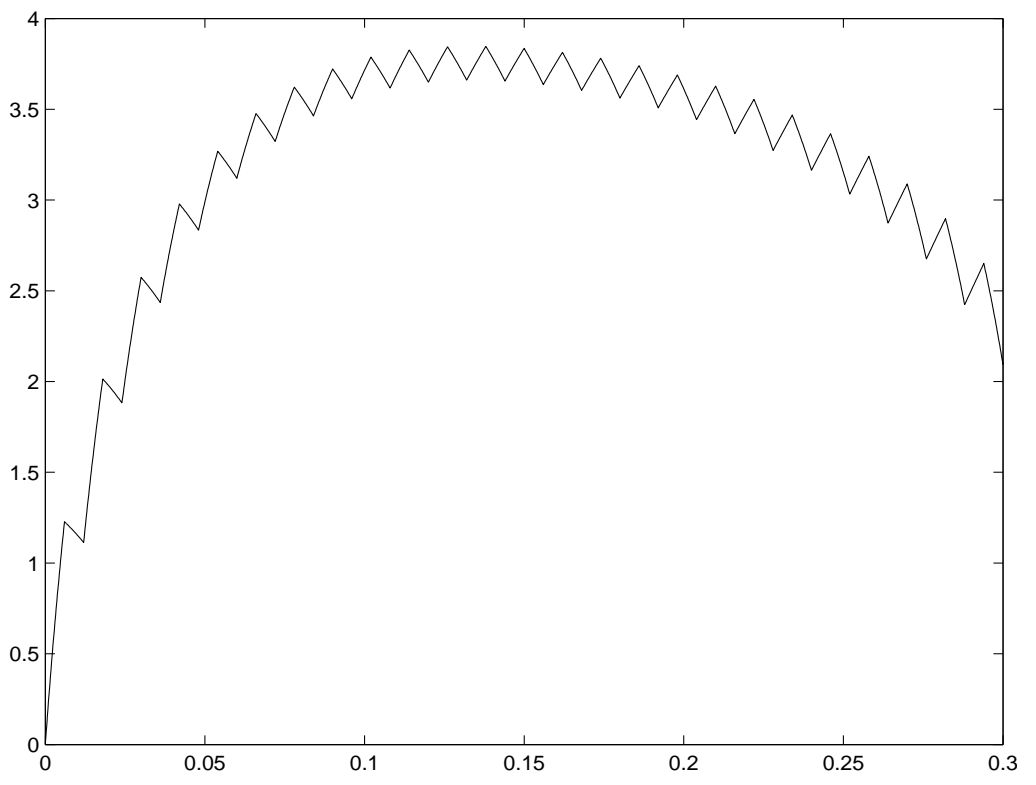

Figure 10. $s(t)$.

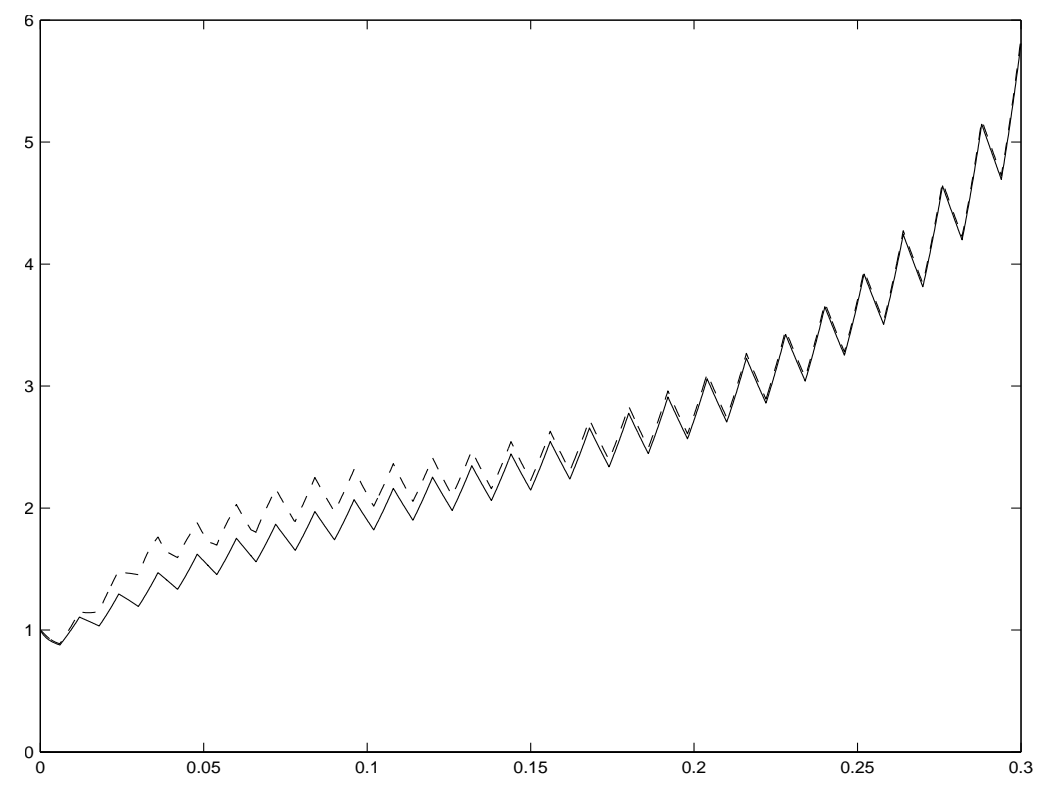

FIGURE 11. $x(t)$.

such that $(k-N) \Delta t \leq t_{1}<t_{2}<\cdots<t_{n} \leq k \Delta t$ and each $s\left(t_{j}\right)$ is of the form $s_{\min }+p \Delta s$. Let us explain more precisely how we build the list (7.15). Let us assume that we have already build the list from $t_{1}$ to $t_{j}$ considering measurements from time $(k-N) \Delta t$ to time $(k-i) \Delta t$. Then between time $(k-i) \Delta t$ and $(k-i+1) \Delta t, l$ and $r$ 
are such that

$$
\begin{aligned}
s_{\text {min }}+l \Delta s<s((k-i) \Delta t) \leq s_{\min }+(l+1) \Delta s & \leq s_{\min }+(l+2) \Delta s \leq \cdots \\
& \leq s_{\min }+(l+r) \Delta s \leq s((k-i+1) \Delta t) \leq s_{\min }+(l+r+1) \Delta s
\end{aligned}
$$

Now we interpolate $t_{j+1}, \ldots, t_{j+r}$ so that $s\left(t_{j+p}\right) \approx s_{\min }+(l+p) \Delta s, p=1, \ldots, r$ and we add $s\left(t_{j+1}\right), \ldots$, $s\left(t_{j+r}\right)$ to the list.

The list (7.15) is then used to look for values appearing at least twice and to estimate $X_{0}$ and $\mu$ at sample values $s_{\min }+(l+p) \Delta s$.

Nevertheless, in order to give some weight to a priori knowledge of $\mu$ and to increase robustness with respect to measurement noise, we do not modify completely $\mu(s)$ when a new value is provided by equations (7.9) and (7.10) and the previous algorithm. In fact, we use a first order filter to actualize $\widehat{\mu_{t}}$, that is, if a new $\mu_{t}(s)$ is obtained from (7.10) at time $t$ for a discretized $s$, we modify $\widehat{\mu_{t}}(s)$ using

$$
\widehat{\mu_{t}}(s)=(1-\beta) \widehat{\mu_{t}}(s)+\beta \mu_{t}(s) .
$$

Despite this filtering, both on measurement and actualization of $\mu$, this algorithm looks rather sensitive to noise. Here, we illustrate our approach without adding any noise on the output $s$.

Figure 5 shows $D(t)$ which is the control. Figures 6 to 9 show the estimation $\widehat{\mu_{t}}(s)$ of $\mu(s)$ at different times. At the beginning of the simulation, we set $\widehat{\mu_{0}}$ to be the Monod law, although the actual unknown law $\mu(s)$ is the Haldane law. Thin lines represent the actual Haldane growth function. Thick lines and dots represent the estimation of the function $\mu$.

At each sample time, as already mentioned, we use the bioreactor model with $\widehat{\mu}_{t}(s)$ as growth function to estimate $x(t)$ using a linear Kalman filter. At the beginning of our simulation, since $\widehat{\mu_{0}}$ is wrong (Monod law instead Haldane law), our observer does not estimate $x$ accurately and there is a bias between the actual $x(t)$ and its estimate $\widehat{x}(t)$. It is expected that when $\mu$ is correctly identified, the observer gives an unbiased estimation of $x$. Indeed, at time 0.15 approximately, $s(t)$ begins to decrease (Fig. 10) and then visits again a domain where $\mu$ has already been identified. Therefore $\mu(s) \approx \widehat{\mu_{t}}(s)$ for $t>0.15$. It is then expected that after time 0.15 , estimation of $x(t)$ will be unbiased. This actually happens, see Figure 11.

\section{A. Appendix}

\section{A.1. A crucial lemma}

\section{Notations:}

1. In this section, we keep the notation $\wedge$ for the exterior product of differential forms on $X$ or on $X \times I$, and, for $V_{1}, V_{2} \in \mathbb{R}^{2}$, we denote by $V_{1} \bar{\wedge} V_{2}$ the determinant of the $2 \times 2$ matrix formed by the vectors $V_{1}, V_{2}$.

2. Again, in this section, for a smooth function $f$ of two variables $(x, \varphi), \quad x \in \mathbb{R}^{n}, \varphi \in \mathbb{R}^{p}$, we denote by $d_{x} f$ $\left(\operatorname{resp} d_{\varphi} f\right)$, the differentials with respect to the $x$ variable (resp. $\varphi$ variable) only.

3. For $x=\left(x_{1}, \ldots, x_{n}\right) \in \mathbb{R}^{n}$, we denote by $\underline{x_{p}} \in \mathbb{R}^{p}$ the vector $\underline{x_{p}}=\left(x_{1}, \ldots, x_{p}\right)$. For a function $h: \mathbb{R}^{n} \rightarrow \mathbb{R}$ of the variables $x_{1}, \ldots, x_{n}$, the notation $\widehat{h^{i_{1}, \ldots, i_{p}}}$ means that $h$ does not depend on $x_{i_{1}}, \ldots, x_{i_{p}}$.

Lemma 7. Consider $h: U \times I \rightarrow \mathbb{R}^{2}, U$ an open connected subset of $\mathbb{R}^{n}$, with a given $C^{\omega}$ coordinate system $x=\left(x_{1}, \ldots, x_{n}\right)$, and $h(x, \varphi)=\left(h_{1}(x, \varphi), h_{2}(x, \varphi)\right)$, such that $d_{\varphi} h$ never vanishes on $U \times I$, and the equation

$$
d_{x} h(x, \varphi) \xi+d_{\varphi} h(x, \varphi) \eta=0, \quad \text { with }\left(\xi_{1}, \ldots, \xi_{p}\right)=0, \quad p<n
$$

has no smooth solution $(\eta, \varphi)(x, \xi)$, on any open subset of $U \times \mathbb{R}^{n-p} \subset\left\{(x, \xi) \mid\left(\xi_{p+1}, \ldots, \xi_{n}\right) \neq 0\right\}$.

Then, there is $Z \subset U$, a closed subanalytic subset of codimension 1 , such that, for all $x_{0} \in U \backslash Z$, it does exist a neighborhood $V_{x_{0}}$ of $x_{0}$ and coordinates $\tilde{x}=\left(\tilde{x}_{1}, \ldots, \tilde{x}_{n}\right)$ on $V_{x_{0}}$, with $\tilde{x}_{1}=x_{1}, \ldots, \tilde{x}_{p}=x_{p}$, and: 
for all $\varphi_{0} \in I$, there is a neighborhood $V_{\varphi_{0}}$ of $\varphi_{0}$, a $C^{\omega}$ real function $\Phi_{\varphi_{0}}$, with open domain $D$ in $V_{x_{0}} \times \Theta_{1} \times \Theta_{2}$, $\Theta_{1}, \Theta_{2}$ open subsets of $\mathbb{R}$, with:

$$
(F) \tilde{x}_{p+1}=\Phi_{\varphi_{0}}\left(\underline{\tilde{x}_{p}}, h_{1}(\tilde{x}, \varphi), h_{2}(\tilde{x}, \varphi)\right),
$$

for all $(\tilde{x}, \varphi) \in V_{x_{0}} \times V_{\varphi_{0}}$.

Moreover,

$$
\begin{gathered}
\text { (G) }\left(\frac{\partial \Phi_{\varphi_{0}}}{\partial h_{1}}, \frac{\partial \Phi_{\varphi_{0}}}{\partial h_{2}}\right) \text { never vanishes on } D, \\
(H) \frac{\partial h}{\partial \varphi} \bar{\wedge} \frac{\partial h}{\partial \tilde{x}_{p+1}} \text { never vanishes on }(U \backslash Z) \times I .
\end{gathered}
$$

Remark 9. $(G)$ is a consequence of $(F)$, since $\tilde{x}$ is a $C^{\omega}$ coordinate system on $X$.

Proof of Lemma 7. Proof for $p+1=n$ : let $E=\left\{(x, \varphi) \mid \frac{\partial h}{\partial \varphi} \bar{\wedge} \frac{\partial h}{\partial x_{n}}(x, \varphi)=0\right\}$, and let $\pi_{E}: E \rightarrow U$. By Hardt's theorem on the stratification of proper subanalytic mappings between subanalytic sets, if $\pi_{E}$ contains an open set, then, there is, on a (may be smaller) open set $\Theta$, a smooth $\left(C^{\omega}\right)$ function $\hat{\varphi}: \Theta \rightarrow E .^{4}$

Hence, $\frac{\partial h}{\partial \varphi} \bar{\wedge} \frac{\partial h}{\partial x_{n}}(x, \hat{\varphi}(x))=0$, for $x \in \Theta$. We chose $x_{0} \in \Theta, \varphi_{0}=\hat{\varphi}\left(x_{0}\right)$.

If $d_{\varphi} h_{1}\left(x_{0}, \varphi_{0}\right) \neq 0$ (we can assume this by the statement of the lemma, eventually changing $h_{1}$ for $h_{2}$ ), then, set:

$$
\hat{\eta}(x, \xi)=-\frac{\left(d_{x} h_{1}\right)(x, \hat{\varphi}(x)) \xi}{\left(d_{\varphi} h_{1}\right)(x, \hat{\varphi}(x))}, \text { for } \xi=\left(0, \ldots, 0, \xi_{n}\right) \neq 0 .
$$

Then the couple $(\hat{\eta}, \hat{\varphi})\left(x, \xi_{n}\right)$ solves equation (A.1). This is a contradiction. Therefore, $Z=\pi_{E}$ has codimension 1 , and on $(U \backslash Z \times I), \frac{\partial h}{\partial \varphi} \bar{\wedge} \frac{\partial h}{\partial x_{n}}$ never vanishes by construction. This proves $(H)$.

Now, $\left(x_{1}, \ldots, x_{p}, h_{1}, h_{2}\right)$ is a coordinate system over small open subsets of $(U \backslash Z) \times I$ :

$$
d x_{1} \wedge \ldots \wedge d x_{p} \wedge d h_{1} \wedge d h_{2}=d x_{1} \wedge \ldots \wedge d x_{p} \wedge d \varphi \wedge d x_{n}\left(\frac{\partial h}{\partial \varphi} \wedge \frac{\partial h}{\partial x_{n}}\right) \neq 0 .
$$

Hence, on such a small open set, $x_{p+1}=\Phi\left(x_{1}, \ldots, x_{p}, h_{1}, h_{2}\right)$.

Proof for $p+1<n$ : There exists $i>p$ such that $\frac{\partial h}{\partial \varphi} \pi \frac{\partial h}{\partial x_{i}}$ does not vanish identically: were it otherwise, for $\xi \neq 0, \xi_{1}=0, \ldots, \xi_{p}=0$, and in a neighborhood of $(x, \varphi)$ such that $d_{\varphi} h_{1} \neq 0, \hat{\eta}=-\frac{d_{x} h_{1} \cdot \xi}{d \varphi h_{1}}$ solves equation (A.1), which is impossible.

Now, for $\xi \neq 0$, for $\xi_{1}=0, \ldots, \xi_{p}=0$,

$$
\begin{aligned}
& \text { (1) } \quad d_{x} h . \xi \bar{\wedge} d_{\varphi} h(x, \varphi)=0 \text { implies: } \\
& \text { (2) } d \varphi\left(d_{x} h . \xi \wedge d_{\varphi} h(x, \varphi)\right)=0 .
\end{aligned}
$$

Indeed, if it is not true, by the implicit function theorem, one can solve (A.3, 1) with respect to $\varphi$, and obtain a smooth solution $\hat{\varphi}(x, \xi)$, on some open set. Setting

$$
\hat{\eta}(x, \xi)=-\frac{\left(d_{x} h_{1}\right)(x, \hat{\varphi}(x, \xi)) \xi}{\left(d_{\varphi} h_{1}\right)(x, \hat{\varphi}(x, \xi))}
$$

we solve again equation (A.1) on an open set, a contradiction.

Statement (A.3) can be rewritten:

$$
\sum_{i=p+1}^{n}\left(d_{x_{i}} h \bar{\wedge} d_{\varphi} h(x, \varphi)\right) \xi_{i}=0 \Rightarrow \sum_{i=p+1}^{n} d_{\varphi}\left(d_{x_{i}} h \bar{\wedge} d_{\varphi} h(x, \varphi)\right) \xi_{i}=0 .
$$

\footnotetext{
${ }^{4}$ Here, in fact, Sard's theorem plus the implicit function theorem is enough to obtain this function $\hat{\varphi}$. But Hardt's theorem is more explicit, and we crucially use subanalyticity elsewhere.
} 
But, one of the $d_{x_{i}} h \bar{\wedge} d_{\varphi} h(x, \varphi)$ does not vanish identically. Hence, in a neighborhood $V$ of some $\left(x_{0}, \varphi_{0}\right)$, we have, for all $i=p+1, \ldots, n$, and for a certain analytic function $\lambda(x, \varphi)$ :

$$
d_{\varphi}\left(d_{x_{i}} h \bar{\wedge} d_{\varphi} h(x, \varphi)\right)=\lambda(x, \varphi)\left(d_{x_{i}} h \bar{\wedge} d_{\varphi} h(x, \varphi)\right),
$$

and then, integrating this (linear) differential equation in $\varphi$ :

$$
d_{x_{i}} h \bar{\wedge} d_{\varphi} h(x, \varphi)=\Lambda(x, \varphi) \omega_{i}(x), \Lambda(x, \varphi) \neq 0, i=p+1, \ldots, n .
$$

This implies, on $V$ :

$$
\omega_{j}(x) d_{x_{i}} h \bar{\wedge} d_{\varphi} h(x, \varphi)-\omega_{i}(x) d_{x_{j}} h \bar{\wedge} d_{\varphi} h(x, \varphi)=0,
$$

for all $i, j>p$. Hence, since $d_{\varphi} h$ never vanishes,

$$
\omega_{j}(x) d_{x_{i}} h-\omega_{i}(x) d_{x_{j}} h=\lambda_{i, j}(x, \varphi) d_{\varphi} h, i, j>p .
$$

This is true again on $V$, and for analytic functions $\omega_{i}(x), \lambda_{i, j}(x, \varphi)$. Moreover, one of the functions $\omega_{i}(x)$ does not vanish $\left(\omega_{p+1} \neq 0\right.$, say): remember that $\omega_{i}\left(x_{0}\right)=d_{x_{i}} h \bar{\wedge} d_{\varphi} h\left(x_{0}, \varphi_{0}\right)$, which is nonzero for some $i$.

Taking $i=p+1, j=p+2$, this implies in particular that $h$ is a ( $\mathbb{R}^{2}$-valued) first integral of the vector field:

$$
(\vec{C}) \varpi_{p+2}(x) \frac{\partial}{\partial x_{p+1}}+\frac{\partial}{\partial x_{p+2}}+\bar{\lambda}(x, \varphi) \frac{\partial}{\partial \varphi} .
$$

This is true on $V$, and for certain analytic functions $\varpi_{p+2}(x), \bar{\lambda}(x, \varphi)$.

The flow of the "characteristic vector field" $\vec{C}$ is:

$$
\exp (t \vec{C})(x)=\left(x_{1}, \ldots, x_{p}, R_{p+2}(t, x), t+x_{p+2}, x_{p+3}, \ldots, x_{n}, S_{p+2}(t, x, \varphi)\right),
$$

with $\frac{\partial R_{p+2}}{\partial x_{p+1}} \neq 0, \frac{\partial S_{p+2}}{\partial \varphi} \neq 0$. Therefore, $h\left(\exp (t \vec{C})(x)=h(x)\right.$, and, setting $\left(x_{p+2}:=0 ; t:=x_{p+2}\right)$, we get:

$$
h\left(x_{1}, \ldots, x_{p}, \bar{R}_{p+2}(x), x_{p+2}, \ldots, x_{n}, \bar{S}_{p+2}(x, \varphi)\right)=h\left(\widehat{x^{p+2}}, \varphi\right),
$$

for some analytic functions $\bar{R}_{p+2}(x), \bar{S}_{p+2}(x, \varphi)$, with $\frac{\partial \bar{R}_{p+2}}{\partial x_{p+1}} \neq 0, \frac{\partial \bar{S}_{p+2}}{\partial \varphi} \neq 0$. Hence, using the implicit function theorem:

$$
h(x, \varphi)=h\left(x_{1}, \ldots, x_{p}, R_{p+2}^{*}(x), 0, x_{p+3}, \ldots, x_{n}, S_{p+2}^{*}(x, \varphi)\right),
$$

or, setting $\tilde{x}_{p+1}=R_{p+2}^{*}(x), \tilde{x}_{i}=x_{i}$ for $i \neq p+1$,

$$
h(\tilde{x}, \varphi)=H\left(\widetilde{x^{p+2}}, S_{p+2}^{*}(\tilde{x}, \varphi)\right), \frac{\partial S_{p+2}^{*}}{\partial \varphi} \neq 0 .
$$

Let us denote these new coordinates $\tilde{x}$ by $x$. We get:

$$
h(x, \varphi)=H\left(x^{\widehat{p+2}}, S_{p+2}(x, \varphi)\right), \frac{\partial S_{p+2}}{\partial \varphi} \neq 0 .
$$

Again (for the same reason as above), there is $i>p$ such that $d_{x_{i}} h \bar{\wedge} d_{\varphi} h(x, \varphi)$ does not vanish identically. Let us assume $d_{x_{p+1}} h \bar{\wedge} d_{\varphi} h(x, \varphi) \neq 0$. Hence $\left(d_{x_{p+1}} H+d_{S} H \frac{\partial S_{p+2}}{\partial x_{p+1}}\right) \bar{\wedge} d_{S} H \frac{\partial S_{p+2}}{\partial \varphi} \neq 0$, which implies $d_{x_{p+1}} H \bar{\wedge} d_{S} H \neq 0$.

Also for the same reason as above, we obtain (A.4), for $i=p+1, j>p+2$, and dividing by $\omega_{i}(x) \neq 0$ :

$$
\omega_{j}(x) d_{x_{p+1}} h-d_{x_{j}} h=\lambda_{p+1, j}(x, \varphi) d_{\varphi} h,
$$


which gives:

$$
\begin{aligned}
\omega_{j}(x) d_{x_{p+1}}(H(x, S(x, \varphi)))-d_{x_{j}}(H(x, S(x, \varphi))) & =\lambda_{p+1, j}(x, \varphi) d_{\varphi}(H(x, S(x, \varphi))), \\
d_{\varphi} S & \neq 0 .
\end{aligned}
$$

or:

$$
\omega_{j}(x)\left(d_{x_{p+1}} H\right)(x, \theta)-\left(d_{x_{j}} H\right)(x, \theta)=\bar{\lambda}_{p+1, j}(x, \theta)\left(d_{\theta} H\right)(x, \theta) .
$$

For $j=p+3$, with the same reasoning as above, we get:

$$
H\left(x_{1}, \ldots x_{p}, \bar{R}_{p+3}(x), x_{p+3}, \ldots, x_{n}, \bar{S}_{p+3}(x, \varphi)\right)=H\left(x^{p+2, p+3}, \varphi\right),
$$

with $\frac{\partial \bar{R}_{p+3}(x)}{\partial x_{p+1}} \neq 0, \frac{\partial S_{p+3}(x)}{\partial \varphi} \neq 0$, or:

$$
H\left(x_{1}, \ldots x_{p}, x_{p+1}, x_{p+3}, \ldots, x_{n}, \varphi\right)=H\left(x_{1}, \ldots x_{p}, R_{p+3}^{*}(x), x_{p+4}, \ldots, x_{n}, S_{p+3}^{*}(x, \varphi)\right) .
$$

Making the change of coordinates $x_{p+1}:=R_{p+3}^{*}(x)$, we get:

$$
H(x, \varphi)=H\left(x_{1}, \ldots x_{p}, x_{p+1}, x_{p+4}, \ldots, x_{n}, S_{p+3}^{*}(x, \varphi)\right) .
$$

At the end, iterating the process, we get that:

$$
h(x, \varphi)=H\left(x_{1}, \ldots, x_{p+1}, S(x, \varphi)\right), \quad \frac{\partial S}{\partial \varphi} \neq 0,
$$

on some open subset of $U \times I$. The coordinates $x_{i}, i=1, \ldots, p$, are unchanged.

As a consequence, we finally get that there is an open dense subanalytic subset $U \backslash Z$ of $U$, and for each $x_{0} \in U \backslash Z$, a coordinate neighborhood of $x_{0},\left(U_{x_{0}}, x\right)$, with coordinates $x_{i}, i=1, \ldots, p$, unchanged, and with:

$$
d h_{1} \wedge d h_{2} \wedge d x_{1} \wedge \ldots \wedge d x_{p+1} \equiv 0
$$

identically on $U_{x_{0}} \times I$, by analyticity.

Now, let $E_{x_{0}}=\left\{(x, \varphi) \mid d h_{1} \wedge d h_{2} \wedge d x_{1} \wedge \ldots \wedge d x_{p}=0\right\}$, and let $\pi E_{x_{0}}$ be the canonical projection of $E_{x_{0}}$ on $U_{x_{0}}$. If $\pi E_{x_{0}}$ contains an open set, again by Hardt's theorem on stratification of proper subanalytic maps, we can find another open subset $\Theta$ of $U$, and a smooth mapping $\hat{\varphi}: \Theta \rightarrow E_{x_{0}}$. Then:

$$
\left(d h_{1} \wedge d h_{2} \wedge d x_{1} \wedge \ldots \wedge d x_{p}\right)_{\mid(x, \hat{\varphi}(x))}=0,
$$

and, in particular, for $j>p,\left(\frac{\partial h_{1}}{\partial x_{j}} \frac{\partial h_{2}}{\partial \varphi}-\frac{\partial h_{1}}{\partial \varphi} \frac{\partial h_{2}}{\partial x_{j}}\right)_{\mid(x, \hat{\varphi}(x))}=0$. Therefore, since $d_{\varphi} h$ is nonzero, equation (A.1) can still be solved, in the following way:

$$
\eta=-\frac{d_{x} h_{1}(x, \hat{\varphi}(x)) \xi}{d_{\varphi} h_{1}(x, \hat{\varphi}(x))}
$$

for $\xi \neq 0$ (if $d_{\varphi} h_{1}\left(x_{0}, \hat{\varphi}\left(x_{0}\right)\right) \neq 0$, and using $h_{2}$ if not).

This contradicts the assumptions of the lemma. Hence, there is a codimension 1 subanalytic closed subset of $U$, called again $Z$, such that, over $U \backslash Z$, each $x_{0}$ has a coordinate neighborhood $\left(U_{x_{0}}, x\right), x_{i}, i=1, \ldots, p$, unchanged, where $d h_{1} \wedge d h_{2} \wedge d x_{1} \wedge \ldots \wedge d x_{p}$ never vanishes, and $d h_{1} \wedge d h_{2} \wedge d x_{1} \wedge \ldots \wedge d x_{p+1}$ is everywhere zero, and this is true over $U_{x_{0}} \times I$.

Since (if $\left.d_{\varphi} h_{1} \neq 0\right), d x_{1} \wedge \ldots \wedge d x_{n} \wedge d h_{1} \neq 0, d h_{1} \wedge d h_{2} \wedge d x_{1} \wedge \ldots \wedge d x_{p+1} \equiv 0$ implies:

$$
h_{2}=H_{2}\left(h_{1}, x_{1}, \ldots, x_{p+1}\right) .
$$


The condition $d h_{1} \wedge d h_{2} \wedge d x_{1} \wedge \ldots \wedge d x_{p} \neq 0$ can be rewritten $\frac{\partial H_{2}}{\partial x_{p+1}} \neq 0$. Hence:

$$
d_{\varphi} h \bar{\wedge} d x_{p+1} h=d_{\varphi} h_{1} \cdot d_{x_{p+1}} H_{2} \neq 0 .
$$

This is $(H)$, in (A.2).

Now, since $\frac{\partial H_{2}}{\partial x_{p+1}} \neq 0$,

$$
\begin{aligned}
& d h_{1} \wedge d h_{2} \wedge d x_{1} \wedge \ldots \wedge d x_{p} \wedge d x_{p+2} \wedge \ldots \wedge d x_{n} \\
& =\left(d_{\varphi} h \wedge d x_{p+1} h\right) d \varphi \wedge d x_{p+1} \wedge d x_{1} \wedge \ldots \wedge d x_{p} \wedge d x_{p+2} \wedge \ldots \wedge d x_{n} \neq 0 .
\end{aligned}
$$

This shows that $h_{1}, h_{2}, x_{1}, \ldots, x_{p}, x_{p+2}, \ldots, x_{n}$ is a coordinate system on some neighborhood $U_{x_{0}, \varphi_{0}} \times V_{\varphi_{0}}$, for all $\varphi_{0} \in I$, and then, since $d h_{1} \wedge d h_{2} \wedge d x_{1} \wedge \ldots \wedge d x_{p+1}$ is identically zero,

$$
x_{p+1}=\Phi_{\varphi_{0}}\left(h_{1}, h_{2}, \underline{x_{p}}\right) .
$$

Now, $I$ being compact, we may cover $\left\{x_{0}\right\} \times I \subset X \times I$ by a finite number of such open neighborhoods $U_{x_{0}, \varphi_{0}} \times V_{\varphi_{0}}$, on which $(F),(G),(H)$ are satisfied. Hence the neighborhood $U_{x_{0}}$ can be taken fixed, independantly of $\varphi_{0}$. This ends the proof.

We thank Jean-Luc Gouzé, from INRIA Sophia-Antipolis, for stating this problem, and for fruitful discussions.

We thank Witold Respondek for the great work he did when reading the paper, and for several improvements he suggested..

\section{REFERENCES}

[1] R. Abraham and J. Robbin, Transversal mappings and flows. W.A. Benjamin Inc. (1967).

[2] G. Bastin and D. Dochain, Adaptive control of bioreactors. Elsevier (1990).

[3] E. Busvelle and J.-P. Gauthier, High-Gain and Non High-Gain Observers for nonlinear systems, edited by Anzaldo-Meneses, Bonnard, Gauthier and Monroy-Perez. World Scientific, Contemp. Trends Nonlinear Geom. Control Theory (2002) 257-286.

[4] J.-P. Gauthier, H. Hammouri and S. Othman, A simple observer for nonlinear systems. Applications to Bioreactors. IEEE Trans. Automat. Control 37 (1992) 875-880.

[5] J.-P. Gauthier and I. Kupka, Observability and observers for nonlinear systems. SIAM J. Control 32 (1994) 975-994.

[6] J.-P. Gauthier and I. Kupka, Observability for systems with more outputs than inputs. Math. Z. 223 (1996) 47-78.

[7] J.-P. Gauthier and I. Kupka, Deterministic Observation Theory and Applications. Cambridge University Press (2001).

[8] M. Goresky and R. Mc Pherson, Stratified Morse Theory. Springer Verlag (1988).

[9] R. Hardt, Stratification of real analytic mappings and images. Invent. Math. 28 (1975) 193-208.

[10] H. Hironaka, Subanalytic Sets, in Number Theory, Algebraic Geometry and Commutative Algebra, in honor of Y. Akizuki. Kinokuniya, Tokyo (1973) 453-493.

[11] M.W. Hirsch, Differential Topology. Springer-Verlag, Grad. Texts in Math. (1976).

[12] R.M. Hirschorn, Invertibility of control systems on Lie Groups. SIAM J. Control Optim. 15 (1977) 1034-1049.

[13] R.M. Hirschorn, Invertibility of Nonlinear Control Systems. SIAM J. Control Optim. 17 (1979) 289-297.

[14] P. Jouan and J.-P. Gauthier, Finite singularities of nonlinear systems. Output stabilization, observability and observers. $J$. Dynam. Control Systems 2 (1996) 255-288.

[15] W. Respondek, Right and Left Invertibility of Nonlinear Control Systems, edited by H.J. Sussmann. Marcel Dekker, New-York, Nonlinear Control. Optim. Control (1990) 133-176.

[16] M. Shiota, Geometry of Subanalytic and Semi-Algebraic Sets. Birkhauser, P.M. 150 (1997).

[17] H.J. Sussmann, Some Optimal Control Applications of Real-Analytic Stratifications and Desingularization, in Singularities Symposium Lojiasiewicz 70, Vol. 43. Banach Center Publications (1998). 